Mon. Not. R. Astron. Soc. 000, ??-?? (2014) $\quad$ Printed 9 April $2019 \quad$ (MN LATEX style file v2.2)

\title{
Chemical analysis of CH stars - III: atmospheric parameters and elemental abundances
}

\author{
Meenakshi Purandardas ${ }^{1,2}$, Aruna Goswami ${ }^{1 \star}$, Partha Pratim Goswami ${ }^{1}$, \\ Shejeelammal J1 ${ }^{1}$ Thomas Masseron ${ }^{3,4}$ \\ ${ }^{1}$ Indian Institute of Astrophysics, Koramangala, Bangalore 560034, India; aruna@iiap.res.in \\ 2 Department of physics, Bangalore university, Jnana Bharathi Campus, Karntaka 560056, India \\ 3 Instituto de Astrofisica de Canarias, E-38205 La Laguna, Tenerife, Spain \\ ${ }^{4}$ Departamento de Astrofisica, Universidad de La Laguna, E-38206 La Laguna, Tenerife, Spain.
}

Accepted —; Received —; in original form -

\begin{abstract}
Elemental abundances of $\mathrm{CH}$ stars can provide observational constraints for theoretical studies on the nucleosynthesis and evolution of low- and intermediate-mass stars. However, available abundance data in literature are quite scanty. In our pursuit to generate a homogeneous database of elemental abundances of $\mathrm{CH}$ stars we have performed a detailed chemical abundance study for a sample of 12 potential $\mathrm{CH}$ star candidates based on high resolution spectroscopy. We present first time abundance analysis for the objects HE0308-1612, CD-281082, HD30443, and HD87853. For the other objects, although limited information is available, detailed chemical composition studies are missing. Our analysis shows $\mathrm{CD}-281082$ to be a very metal-poor object with $[\mathrm{Fe} / \mathrm{H}]=-2.45$ and enriched in carbon with $[\mathrm{C} / \mathrm{Fe}]=2.19$. With a ratio of $[\mathrm{Ba} / \mathrm{Eu}] \sim 0.02$ the star satisfies the classification criteria of a CEMP-r/s star. The objects $\mathrm{CD}-382151$ with $[\mathrm{Fe} / \mathrm{H}]=-2.03$ and $\mathrm{HD} 30443$ with $[\mathrm{Fe} / \mathrm{H}] \sim-1.68$ are found to show the characteristic properties of CH stars. HE0308-1612 and HD87853 are found to be moderately metal-poor with $[\mathrm{Fe} / \mathrm{H}] \sim-0.73$; while $\mathrm{HE} 0308-1612$ is moderately enhanced with carbon $([\mathrm{C} / \mathrm{Fe}] \sim 0.78)$ and shows the spectral properties of $\mathrm{CH}$ stars, the abundance of carbon could not be estimated for HD87853. Among the two moderately metal-poor objects, HD87080 $([\mathrm{Fe} / \mathrm{H}]=-0.48)$ shows near solar carbon abundance, and HD176021 $([\mathrm{Fe} / \mathrm{H}]=-0.63)$ is mildly enhanced in carbon with $[\mathrm{C} / \mathrm{Fe}]=0.52$. HD176021 along with HD202020 a known binary, exhibit the characteristic properties of $\mathrm{CH}$ stars as far as the heavy element abundances are concerned. Five objects in our sample show spectral properties that are normally seen in barium stars.
\end{abstract}

Key words: stars: abundances - stars: chemically peculiar - stars: carbon

\section{INTRODUCTION}

In a recent study by Cristallo et al. (2016), a homogeneous set of abundance data from CH stars (Goswami et al. 2006; Goswami, Karinkuzhi \& Shantikumar 2010; Karinkuzhi \& Goswami 2014, 2015; Goswami, Aoki \& Karinkuzhi 2016) are used in an attempt to constrain the physics and the nucleosynthesis occurring in low-mass AGB stars. The scope of enhancing such studies based on a larger homogeneous abundance data was highlighted. Particularly, the data corresponding to the lower metallicity regime were found to be scanty. In our efforts to provide tighter observational

\footnotetext{
* E-mail: aruna@iiap.res.in
}

constraints we have undertaken to conduct chemical composition study for a larger sample selected from the $\mathrm{CH}$ star catalogue of Bartkevicius (1996), and our previous work (Goswami 2005, Goswami et al. 2007, 2010). In this work we report results for twelve objects. As will be shown later in detail, we have found two objects to be very metal-poor stars with $[\mathrm{Fe} / \mathrm{H}]<-2$ and enhanced in carbon with $[\mathrm{C} / \mathrm{Fe}]>$ 1. Five objects exhibit characteristic properties of $\mathrm{CH}$ stars, and the other five show spectral properties that are normally seen in barium stars.

$\mathrm{CH}$ stars characterized by iron-deficiency and carbon enhancement (with $-2 \leq[\mathrm{Fe} / \mathrm{H}] \leq-1,[\mathrm{C} / \mathrm{Fe}] \geq 0.7,[\mathrm{Ba} / \mathrm{Fe}]$ $\geq 1.0$ ) are a distinct group of stars (Keenan 1942) that are long been used as halo tracers. CEMP-s stars as defined in Beers \& Christlieb (2005), classified as stars with [C/Fe] > 
1 and $[\mathrm{Fe} / \mathrm{H}]<-2$ are more metal-poor counterparts of $\mathrm{CH}$ stars and believed to be extrinsically enriched with carbon and s-process elements. From observational evidences, $\mathrm{CH}$ stars are known to be binary systems (McClure 1983, 1984, McClure \& Woodsworth 1990), with a now invisible white dwarf companion. Until recently, the CEMP-s stars with enhanced abundances of heavy s-process elements and carbon are also thought to be binary systems (Lucatello et al. 2005, Bisterzo et al. 2011, Starkenburg et al. 2014, Jorissen et al. 2016) and inferred to have a similar origin as that of the $\mathrm{CH}$ stars. However, based on a long-term campaign of precision radial-velocity monitoring of a sample of 22 CEMP-s and CEMP-r/s stars, Hansen et al. (2016) reported 18 of them (82 percent \pm 26 percent) to be bonafide binary systems; the rest are either single or objects with extremely long period. If single, with carbon and barium enhancement these objects draw special attention for an intrinsic origin. Among many scenarios, the ones involving massive stars producing barium and other heavy elements through a weak s-process mechanism are suggested by many authors as possible mechanism(s) (Frischknecht et al. 2016, and references therein). These suggestions however require detailed investigation and larger samples are clearly desirable. The enhanced abundances of heavy elements observed in the (secondary) star of the $\mathrm{CH}$ binary star systems that are in the giant phase of evolution are attributed to a now invisible white dwarf companion (primary) which has evolved through the Asymptotic Giant Branch (AGB) phase. During the AGB phase the heavy elements were synthesized and the synthesized materials are transferred through mass transfer mechanisms to the secondary. Although the mass transfer mechanism(s) are not well understood despite several dedicated studies (e.g., Abate et al. 2013, 2015a,b, and references therein) the surface chemical composition of the secondaries can be used as important diagnostics for s-process nucleosynthesis in the primary.

The $12 \mathrm{CH}$ star candidates analysed in this study cover a wide range in metallicity from near solar to $[\mathrm{Fe} / \mathrm{H}]=-2.4$. While CD-28 1082, and CD-38 2151 are found to be very metal-poor with $[\mathrm{Fe} / \mathrm{H}]<-2.0$, HD 176021 and HD 87080 are found to be mildly metal-poor with metallicities -0.63 and -0.48 respectively. We have investigated anew the elemental abundances of two objects (HD 87080, HD 188985) previously studied by Allen \& Barbuy (2006a) and another two objects (HD 29370, HD 123701) previously studied by de Castro et al. (2016) that allowed us to make a direct comparison with ours and verify our estimates. HD 87080 was also analysed by Pereira \& Junqueira (2003); they found that iron group, $\alpha$-elements, manganese, as well as sodium and aluminium of HD 87080 follow the abundance pattern of the Galactic disk stars. Analysis of North, Berthet \& Lanz (1994a,b) have confirmed HD 188985 to be a barium dwarf. We have also presented abundances for $\mathrm{C}, \mathrm{N}$, and $\mathrm{O}$ for these objects that were not reported in their previous studies.

In section 2, we briefly describe the source of the spectra used in the present study and present estimates of radial velocities. Section 3 gives the estimates of photometric temperature. Section 4 describes the methodology used for determination of atmospheric parameters and estimates of the elemental abundances. This section also provides a discussion on the estimates of stellar masses and age of the pro- gramme stars. A comparison between the estimates obtained from spectra using VBT echelle and FEROS is presented in section 5 . In section 6 we discuss the abundance analysis. In section 7 , we discuss the kinematic analysis. stars are presented in section 8 and conclusions are drawn in section 9 .

\section{OBSERVATIONS AND DATA REDUCTION}

For the objects HD 29370, CD-38 2151, HD 50264, HD 87080, HD 123701, and HD 188985, high resolution $(\lambda / \delta \lambda \sim 72000)$ spectra were obtained during 2018 March May using the high resolution fiber fed Echelle spectrometer attached to the $2.34 \mathrm{~m}$ Vainu Bappu Telescope (VBT) at the Vainu Bappu Observatory (VBO), Kavalur. The wavelength coverage of these spectra spans from 4100 to $9350 \AA$ with gaps between orders. The data-reduction is done following the standard procedures using IRAF $^{1}$ spectroscopic data reduction package. A few high-resolution spectra obtained with the FEROS (Fiber-fed Extended Range Optical Spectrograph) of the $1.52 \mathrm{~m}$ telescope of ESO at La Silla, Chile are also used in the present study. The wavelength coverage of FEROS spectra spans from 3500 - $9000 \AA$ and the spectral resolution is $\sim 48000$. For the objects HD 30443 and HE $0308-1612$, the high resolution $(\lambda / \delta \lambda \sim 60000)$ spectra were obtained during 2017 November and 2018 January using the high-resolution fiber fed Hanle Echelle SPectrograph (HESP) attached to the $2 \mathrm{~m}$ Himalayan Chandra Telescope (HCT) at the Indian Astronomical Observatory, Hanle. The wavelength coverage of these spectra spans from 3530-9970 $\AA$. Table 1 lists the basic data for the programme stars and details of the source of spectra. A few sample spectra are shown in Figure 1.

\section{PHOTOMETRIC TEMPERATURES}

The photometric temperatures of our programme stars are estimated using the calibration equations of Alonso, Arribas \& Martinez-Roger(1999, 2001) and following the detailed procedures as given in our earlier papers (Goswami et al. 2006, 2016). As our programme star sample consists of both main-sequence stars and giants, we have made use of the corresponding calibration equations separately for each group. J, H, K magnitudes of the objects required for temperature estimates are taken from SIMBAD database which came from 2MASS (Cutri et al. 2003). We have used the estimated photometric temperatures as the initial guess for the estimation of spectroscopic temperature of the objects. The estimated photometric temperatures are presented in Table 2 .

1 IRAF is distributed by the National Optical Astronomical Observatories, which is operated by the Association for Universities for Research in Astronomy, Inc., under contract to the National Science Foundation 
Table 1: Basic data for the programme stars

\begin{tabular}{|c|c|c|c|c|c|c|c|c|c|c|}
\hline Star & $\mathrm{RA}(2000)$ & Dec.(2000) & B & $\mathrm{V}$ & $\mathrm{J}$ & $\mathrm{H}$ & $\mathrm{K}$ & $\begin{array}{l}\text { Exposure } \\
\text { (seconds) }\end{array}$ & Date of obs. & $\begin{array}{c}\text { Source } \\
\text { of spectrum }\end{array}$ \\
\hline HE $0308-1612$ & 031027.07 & $\begin{array}{lll}-16 & 00 & 40.1\end{array}$ & 12.7 & - & 10.03 & 9.48 & 9.33 & $2700(4)$ & 24-01-2018 & HESP \\
\hline CD-28 1082 & 031626.43 & -281048.02 & 11.55 & 10.41 & 8.89 & 8.44 & 8.30 & 3600 & 05-01-2000 & FEROS \\
\hline HD 29370 & 043540.04 & $-4200 \quad 05.58$ & 10.43 & 9.33 & 7.57 & 7.08 & 6.97 & $\begin{array}{c}2700 \\
2700(2)\end{array}$ & $\begin{array}{l}04-01-2000 \\
07-03-2018\end{array}$ & $\begin{array}{c}\text { FEROS } \\
\text { VBT Echelle }\end{array}$ \\
\hline HD 30443 & 044916.02 & +35006.49 & 11.2 & 8.82 & 4.62 & 3.28 & 2.83 & $2400(2)$ & $07-11-2017$ & HESP \\
\hline CD-38 2151 & $05 \quad 4001.94$ & -384925.89 & 10.75 & 9.27 & 7.16 & 6.56 & 6.39 & $\begin{array}{c}3600 \\
2700(2)\end{array}$ & $\begin{array}{l}06-01-2000 \\
06-03-2018\end{array}$ & $\begin{array}{c}\text { FEROS } \\
\text { VBT Echelle }\end{array}$ \\
\hline HD 50264 & 065112.49 & -293432.35 & 9.63 & 9.05 & 7.91 & 7.61 & 7.57 & $\begin{array}{c}1200 \\
2700(2)\end{array}$ & $\begin{array}{c}10-11-1999 \\
6-03-2018\end{array}$ & $\begin{array}{c}\text { FEROS } \\
\text { VBT Echelle }\end{array}$ \\
\hline HD 87080 & 100200.85 & -334106.50 & 10.15 & 9.37 & 8.00 & 7.68 & 7.56 & $\begin{array}{c}2100 \\
2700(3)\end{array}$ & $\begin{array}{l}05-01-2000 \\
09-04-2018\end{array}$ & $\begin{array}{c}\text { FEROS } \\
\text { VBT Echelle }\end{array}$ \\
\hline HD 87853 & 100758.78 & +083037.24 & 10.12 & 9.66 & 8.81 & 8.57 & 8.55 & 1800 & $06-01-2000$ & FEROS \\
\hline HD 123701 & 140955.10 & -302633.91 & 9.54 & 8.51 & 6.78 & 6.30 & 6.21 & $\begin{array}{c}1200 \\
2700(3)\end{array}$ & $\begin{array}{l}05-01-2000 \\
06-03-2018\end{array}$ & $\begin{array}{c}\text { FEROS } \\
\text { VBT Echelle }\end{array}$ \\
\hline HD 176021 & 190237.30 & -645531.30 & 8.20 & 7.58 & 6.47 & 6.20 & 6.11 & 300 & $14-07-2000$ & FEROS \\
\hline HD 188985 & 195958.29 & -485831.05 & 9.07 & 8.56 & 7.54 & 7.32 & 7.23 & $\begin{array}{c}600 \\
2700(1)\end{array}$ & $\begin{array}{l}14-07-2000 \\
09-04-2018\end{array}$ & $\begin{array}{c}\text { FEROS } \\
\text { VBT Echelle }\end{array}$ \\
\hline HD 202020 & 211339.47 & -093741.73 & 9.90 & 9.37 & 8.15 & 7.89 & 7.82 & 1200 & $14-07-2000$ & FEROS \\
\hline
\end{tabular}

Note.The numbers in the parenthesis with exposures indicate the number of frames taken.

PIs of the original observing proposals are V. Hill for FEROS 1999 spectra, F. Primas for FEROS 2000 spectra, and A. Goswami for VBT Echelle and HESP spectra.

Table 2 : Temperatures from photometry

\begin{tabular}{|c|c|c|c|c|c|c|c|c|c|c|c|}
\hline Star name & $\begin{array}{l}T_{e} f f \\
(\mathrm{~J}-\mathrm{K})\end{array}$ & $\begin{array}{l}T_{e} f f \\
(-0.05) \\
(\mathrm{J}-\mathrm{H})\end{array}$ & $\begin{array}{l}T_{e} f f \\
(-0.5) \\
(\mathrm{J}-\mathrm{H})\end{array}$ & $\begin{array}{l}T_{e} f f \\
(-1.5) \\
(\mathrm{J}-\mathrm{H})\end{array}$ & $\begin{array}{l}T_{e} f f \\
(-0.05) \\
(\mathrm{V}-\mathrm{K})\end{array}$ & $\begin{array}{l}T_{e} f f \\
(-0.5) \\
(\mathrm{V}-\mathrm{K})\end{array}$ & $\begin{array}{l}T_{e} f f \\
(-1.5) \\
(\mathrm{V}-\mathrm{K})\end{array}$ & $\begin{array}{l}T_{e} f f \\
(-0.05) \\
(\mathrm{B}-\mathrm{V})\end{array}$ & $\begin{array}{l}T_{e} f f \\
(-0.5) \\
(\mathrm{B}-\mathrm{V})\end{array}$ & $\begin{array}{l}T_{e} f f \\
(-1.5) \\
(\mathrm{B}-\mathrm{V})\end{array}$ & $\begin{array}{l}\text { Spectroscopic } \\
\text { estimates }\end{array}$ \\
\hline HE $0308-1612$ & 4420.26 & 4413.24 & 4428.17 & 4461.72 & - & - & - & - & - & - & 4600 \\
\hline $\mathrm{CD}-28 \quad 1082$ & 4862.67 & 5044.69 & 5091.08 & 5119.74 & 4956.30 & 4941.81 & 4928.88 & 4534.58 & 4452.10 & 4353.37 & 5200 \\
\hline HD 29370 & 4830.66 & 4874.24 & 4916.69 & 4941.53 & 4703.69 & 4685.78 & 4663.45 & 4604.81 & 4515.99 & 4406.40 & 4970 \\
\hline HD 30443 & - & - & - & - & - & - & - & - & - & - & 4040 \\
\hline $\mathrm{CD}-382151$ & 4358.16 & 4465.73 & 4499.41 & 4545.89 & 4292.39 & 4275.88 & 4255.08 & 4015.86 & 3975.85 & - & 4600 \\
\hline HD 50264 & 5964.48 & 5890.11 & 5903.72 & 5934.18 & 5778.41 & 5773.23 & 5780.91 & 5892.20 & 5735.39 & 5509.55 & 5900 \\
\hline HD 87080 & 5416.07 & 5704.45 & 5767.58 & - & 5295.93 & 5286.78 & 5288.41 & 5257.63 & 5133.97 & 4934.15 & 5600 \\
\hline HD 87853 & 6361.55 & 6108.46 & 6121.51 & 6150.71 & 6373.71 & 6377.72 & 6410.18 & 6376.35 & 6200.93 & 5953.78 & 6250 \\
\hline HD 123701 & 4928.34 & 4915.63 & 4959.03 & 4984.78 & 4762.04 & 4744.87 & 4724.61 & 5691.86 & 5568.66 & 5378.63 & 5340 \\
\hline HD 176021 & 5780.58 & 6012.43 & 6084.20 & - & 5739.06 & 5738.20 & 5762.19 & 5691.86 & 5568.66 & 5378.63 & 5900 \\
\hline HD 188985 & 6132.50 & 6461.37 & 6473.32 & 6500.05 & 6018.47 & 6016.72 & 6033.72 & 6165.46 & 5998.25 & 5760.42 & 6250 \\
\hline HD 202020 & 5933.00 & 6078.46 & 6152.15 & - & 5628.51 & 5625.44 & 5643.49 & 5978.92 & 5856.51 & 5674.51 & 5960 \\
\hline
\end{tabular}

\section{SPECTROSCOPIC STELLAR PARAMETERS}

Equivalent widths of a set of clean and unblended Fe I and Fe II lines are used for the estimation of atmospheric parameters. The lines are selected to have the excitation potential in the range $0.0-5.0 \mathrm{eV}$ and the equivalent widths in the range $20-180 \mathrm{~mA}$. The number of Fe II lines measured is small in number as it is difficult to get clean Fe II lines in the spectra. We have made use of Local Thermodynamic Equilibrium (LTE) analysis of the measured equivalent widths using a recent version of MOOG of Sneden (1973). Model atmospheres used are selected from Kurucz grid of model atmospheres with no convective overshooting (http://cfaku5.cfa.hardvard.edu/). Solar abundances are adopted from Asplund, Grevesse \& Sauval (2009).

A trend between the iron abundance derived from the Fe I lines and the excitation potential of these lines with a zero slope defines the effective temperature. The microturbulent velocity is taken to be that value for which the abundances derived from the Fe I lines do not show any dependence on the reduced equivalent width $\left(W_{\lambda} / \lambda\right)$. The surface gravity $\log g$ is determined corresponding to the adopted values of the effective temperature and microturbulent velocity for which Fe I and Fe II lines give near equal abundance values. The estimated abundances from Fe I and Fe II lines as a function of excitation potential and equivalent widths, respectively, are shown in figures that are made available as on-line materials. The derived atmospheric parameters are listed in Table 3.

We have estimated the mass of our programme stars from their postion in the HR diagram, $\log \left(L / L_{\odot}\right)$ versus spectroscopic $\mathrm{T}_{\text {eff }}$ plots (Figs 2-4). The visual magnitude $\mathrm{V}$ is taken from SIMBAD and the parallaxes are taken from Gaia (Gaia collaboration 2016, 2018b, https://gea.esac.esa.int/archive/) whenever possible. The bolometric corrections are determined separately for mainsequence and subgiants/giants using the empirical calibrations of Alonso et al.(1999). Interstellar extinction $\left(\mathrm{A}_{v}\right)$ for objects with Galactic latitude $\mathrm{b}<50$ degree are calculated based on formulae by Chen et al (1998). We made use of Girardi et al. (2000) database (http://pleiadi.pd.astro.it/) of evolutionary tracks to estimate the mass of the stars. For near solar objects evolutionary tracks corresponding to $\mathrm{Z}=$ 0.019 are chosen. Surface gravity $\log g$ is calculated from the estimated mass using the relation

$\log \left(\mathrm{g} / \mathrm{g}_{\odot}\right)=\log \left(\mathrm{M} / \mathrm{M}_{\odot}\right)+4 \log \left(\mathrm{T}_{\text {eff }} / \mathrm{T}_{\text {eff } \odot}\right)+0.4\left(\mathrm{M}_{b o l}-\right.$ $\mathrm{M}_{\text {bol } \odot)}$

The adopted values for the Sun are $\log \mathrm{g}_{\odot}=4.44, \mathrm{~T}_{\text {eff } \odot}=$ $5770 \mathrm{~K}$, and $\mathrm{M}_{b o l \odot}=4.75 \mathrm{mag}$ (Yang et al. 2016).

We have also determined the age of our programme 
Table 3: Derived atmospheric parameters and radial velocities of the programme stars.

\begin{tabular}{|c|c|c|c|c|c|c|c|}
\hline Star & $\begin{array}{l}\mathrm{T}_{\text {eff }} \\
(\mathrm{K})\end{array}$ & $\begin{array}{c}\log g \\
\operatorname{cgs}\end{array}$ & $\begin{array}{c}\zeta \\
\left(\mathrm{km} \mathrm{s}^{-1}\right)\end{array}$ & {$[\mathrm{Fe} \mathrm{I} / \mathrm{H}]$} & {$[\mathrm{Fe} \mathrm{II/H}]$} & $\begin{array}{c}\mathrm{V}_{r} \\
\left(\mathrm{~km} \mathrm{~s}^{-1}\right) \\
\text { (our estimates) }\end{array}$ & $\begin{array}{c}\mathrm{V}_{r} \\
\left(\mathrm{~km} \mathrm{~s}^{-1}\right) \\
\text { (from literature) }\end{array}$ \\
\hline HE $0308-1612$ & 4600 & 1.7 & 1.42 & $-0.72 \pm 0.19$ & $-0.73 \pm 0.15$ & $85.5 \pm 1.22(\mathrm{HESP})$ & $76.41 \pm 0.51$ \\
\hline CD-28 1082 & 5200 & 1.90 & 1.42 & $-2.46 \pm 0.08$ & $-2.44 \pm 0.02$ & $-26.7 \pm 0.3$ (FEROS $)$ & - \\
\hline HD 29370 & 4970 & 2.40 & 1.92 & $-0.15 \pm 0.11$ & $-0.13 \pm 0.04$ & $-1.5 \pm 0.03($ FEROS $)$ & $16.0 \pm 2.6$ \\
\hline HD 30443 & 4040 & 2.05 & 2.70 & $-1.68 \pm 0.05$ & $-1.69 \pm 0.11$ & $66.61 \pm 0.20(\mathrm{HESP})$ & $63.17 \pm 0.22$ \\
\hline CD-38 2151 & 4600 & 0.90 & 2.30 & $-2.03 \pm 0.10$ & -2.03 & $\begin{array}{c}138.5 \pm 0.6(\text { FEROS }) \\
139.7 \pm 1.9(\mathrm{VBT})\end{array}$ & 125 \\
\hline HD 50264 & 5900 & 4.60 & 0.62 & $-0.14 \pm 0.09$ & $-0.11 \pm 0.07$ & $\begin{array}{c}65.8 \pm 0.4(\text { FEROS }) \\
67.8 \pm 2.6(\mathrm{VBT})\end{array}$ & $56.9 \pm 0.4$ \\
\hline HD 87080 & 5600 & 3.74 & 1.10 & $-0.47 \pm 0.09$ & $-0.49 \pm 0.04$ & $\begin{array}{c}5.9 \pm 0.02(\text { FEROS }) \\
-1.7 \pm 0.5(\mathrm{VBT})\end{array}$ & $-0.5 \pm 1.4$ \\
\hline HD 87853 & 6250 & 2.5 & 1.63 & $-0.72 \pm 0.09$ & $-0.74 \pm 0.09$ & $-3.35 \pm 0.05$ (FEROS $)$ & - \\
\hline HD 123701 & 5340 & 3.50 & 1.44 & $0.05 \pm 0.09$ & $0.07 \pm 0.09$ & $\begin{array}{c}-18.2 \pm 0.1(\mathrm{FEROS}) \\
-24.8 \pm 0.6(\mathrm{VBT})\end{array}$ & - \\
\hline HD 176021 & 5900 & 3.95 & 1.02 & $-0.62 \pm 0.08$ & $-0.65 \pm 0.05$ & $109.1 \pm 0.5($ FEROS $)$ & $108.4 \pm 0.9$ \\
\hline HD 188985 & 6250 & 4.30 & 0.60 & $0.01 \pm 0.10$ & $0.01 \pm 0.09$ & $6.7 \pm 0.03$ (FEROS) & 8.2 \\
\hline HD 202020 & 5960 & 4.38 & 0.86 & $0.04 \pm 0.09$ & $0.08 \pm 0.03$ & $-18.6 \pm 0.1$ (FEROS $)$ & $-24.5 \pm 0.14$ \\
\hline
\end{tabular}

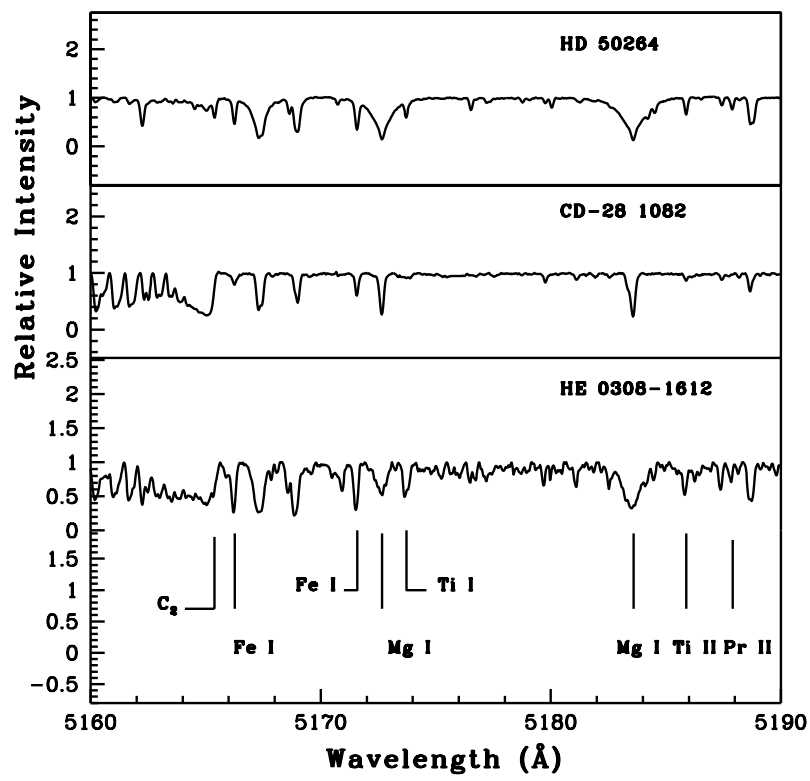

Figure 1. Examples of sample spectra of the programme stars in the wavelength region 5160 to $5190 \AA$. In the upper panel the spectrum of a barium star, in the middle panel the spectrum of a CEMP-r/s star and in the bottom panel the spectrum of a $\mathrm{CH}$ star are shown.

stars from their locations in the HR diagram. We have made use of the Girardi et al. (2000) database of isochrones. We used isochrones corresponding to $\mathrm{z}=0.0004$ for $\mathrm{CD}-28$ 1082, and $\mathrm{z}=0.004$ for HD 176021, HD 87080 and HD 87853. For objects with near solar metallicity, the isochrones corresponding to $\mathrm{z}=0.019$ are adopted. These are illustrated in Figures 5-7. The age estimates are presented in Table 4.

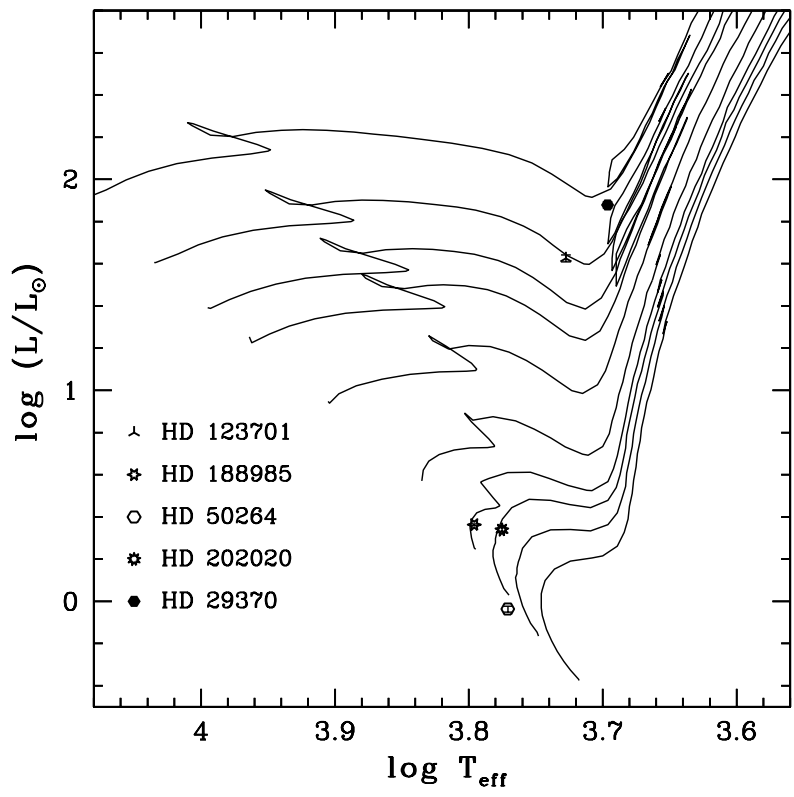

Figure 2. The locations of HD 123701, HD 188985, HD 50264, HD 202020 and HD 29370. The evolutionary tracks for 0.9, 1.0, $1.1,1.2,1.4,1.7,2.0,2.2,2.5$ and $3.0 \mathrm{M}_{\odot}$ are shown from bottom to top.

\section{COMPARISON WITH LITERATURE}

To verify our estimates measured from the VBT spectra we have analyzed anew the FEROS spectra of HD 87080 and HD 188985 which were previously studied by Allen \& Barbuy (2006a,b), and HD 123701 and HD 29370 previously studied by de Castro et al. (2016) and compared their results with those obtained by us from VBT spectra. Our estimates of atmospheric parameters and metallicity for these stars match closely with those obtained by Allen \& Barbuy (2006a,b) and de Castro et al. (2016) within the error limits. As a check to our estimates we have randomly selected a few lines, and performed spectrum synthesis calculation 
Table 4: Estimates of $\log g$ using parallax method

\begin{tabular}{lccccccc}
\hline Star name & $\begin{array}{c}\text { Parallax } \\
\text { (mas) }\end{array}$ & $M_{\text {bol }}$ & $\log \left(\mathrm{L} / \mathrm{L}_{\odot}\right)$ & Mass $\left(\mathrm{M}_{\odot}\right)$ & $\begin{array}{c}\text { log g } \\
(\mathrm{cgs})\end{array}$ & $\begin{array}{c}\text { log g (spectroscopic) } \\
(\mathrm{cgs})\end{array}$ & $\begin{array}{c}\text { Age } \\
(\mathrm{Gyr})\end{array}$ \\
\hline HE 0308-1612 & $0.46 \pm 0.05$ & - & - & - & - & 1.70 & - \\
CD -28 1082 & $1.09 \pm 0.05$ & $0.27 \pm 0.09$ & $1.79 \pm 0.04$ & $1.05 \pm 0.05$ & $2.52 \pm 0.03$ & 1.9 & 7.9 \\
HD 29370 & $1.58 \pm 0.03$ & $0.05 \pm 0.03$ & $1.88 \pm 0.02$ & 2.50 & $2.70 \pm 0.02$ & 2.4 & 0.56 \\
CD-38 2151 & $0.56 \pm 0.02$ & $-2.42 \pm 0.09$ & $2.87 \pm 0.04$ & $0.90 \pm 0.08$ & $1.13 \pm 0.02$ & 0.90 & 6.31 \\
HD 30443 & $2.16 \pm 0.05$ & $0.76 \pm 0.05$ & $2.20 \pm 0.03$ & - & - & 2.05 & - \\
HD 50264 & $15.31 \pm 0.25$ & $4.84 \pm 0.04$ & $-0.04 \pm 0.01$ & 1.10 & $4.57 \pm 0.02$ & 4.6 & 0.16 \\
HD 87080 & $4.58 \pm 0.07$ & $2.54 \pm 0.03$ & $0.88 \pm 0.1$ & $1.30 \pm 0.02$ & $3.62 \pm 0.01$ & 3.7 & 3.16 \\
HD 87853 & $5.05 \pm 0.08$ & $3.10 \pm 0.04$ & $0.66 \pm 0.01$ & 1.05 & $3.95 \pm 0.01$ & 2.50 & 4.7 \\
HD 123701 & $2.94 \pm 0.06$ & $0.69 \pm 0.04$ & $1.62 \pm 0.02$ & $2.50 \pm 0.02$ & $3.08 \pm 0.01$ & 3.5 & 0.59 \\
HD 176021 & $19.59 \pm 0.5$ & $3.94 \pm 0.01$ & $0.32 \pm 0.002$ & 0.85 & $4.08 \pm 0.005$ & 3.9 & 0.1 \\
HD 188985 & $12.79 \pm 0.05$ & $3.84 \pm 0.01$ & $0.36 \pm 0.004$ & 1.20 & $4.38 \pm 0.005$ & 4.3 & 3.98 \\
HD 202020 & $8.30 \pm 0.07$ & $3.89 \pm 0.02$ & $0.34 \pm 0.01$ & 1.10 & $4.19 \pm 0.005$ & 4.4 & 5.0 \\
\hline
\end{tabular}

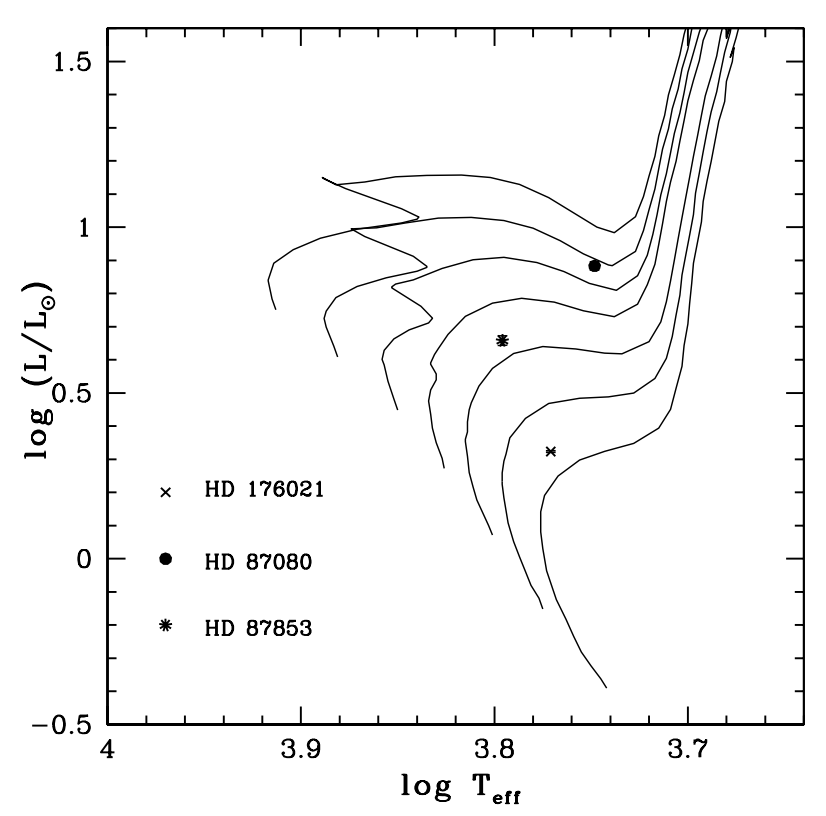

Figure 3. The locations of HD 87080, HD 87853 and HD 176021. The evolutionary tracks for $0.8,0.9,1.0,1.1,1.2,1.3$, and $1.4 \mathrm{M}_{\odot}$ are shown from bottom to top.

using our estimates of atmospheric parameters as well as the parameters obtained by Allen \& Barbuy (2006a) and de Castro et al. (2016) for the respective stars. The fits match quite well. As an example we have shown the best fits obtained in a few cases (Fe I 5434.523 $\AA$, Fe II $4620.521 \AA$ and $\mathrm{Ba}$ II $5853.67 \AA$ ) in Fig. 8. We have adopted our estimates for further analysis of the objects. Table 5 presents a comparison of our estimates with those of Allen \& Barbuy (2006), de Castro et al. (2016) and other literature values. In Fig. 9, we have shown a comparison of VBT Echelle spectrum of CD-38 2151 with the FEROS spectrum. The spectral features match well. Fig. 10 illustrates a comparison of equivalent widths measured in FEROS spectrum with those measured on VBT spectrum; the measurements match closely. Such comparisons were performed for all the stars for which both VBT and FEROS spectra are available; here for illustration purpose we have shown the comparison for one case.

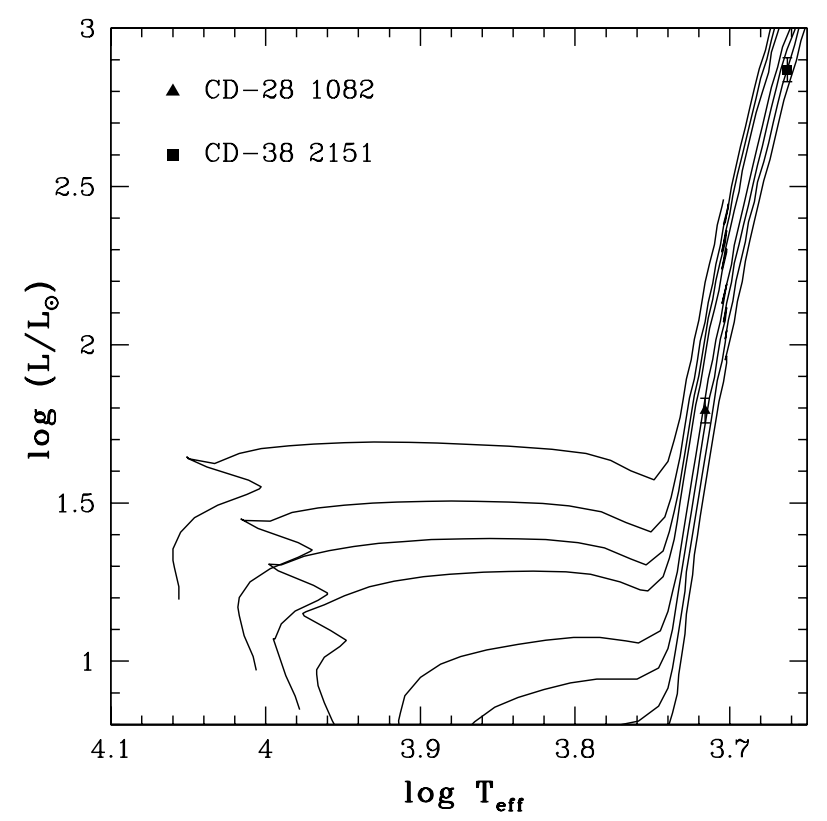

Figure 4. The locations of CD-28 1082 and CD-38 2151. The evolutionary tracks for $0.8,0.9,1.0,1.1,1.3,1.4,1.5$ and $1.7 \mathrm{M}_{\odot}$ are shown from bottom to top.

\section{ABUNDANCE ANALYSIS}

Elemental abundances are measured using equivalent widths of good lines as well as using spectral synthesis calculation whenever applicable. Lines due to different elements are identified by overplotting the Arcturus spectra on the spectra of our programme stars. A master line list with the measured equivalent widths, and line information such as lower excitation potential and log gf values of the lines due to different elements considered for abundance estimates is prepared using the Kurucz database. Only symmetric and clean lines are considered for the measurement of equivalent widths. Abundances of light elements, C, N O, odd-Z element $\mathrm{Na}, \alpha-$ and Fe-peak elements $\mathrm{Mg}, \mathrm{Si}, \mathrm{Ca}, \mathrm{Sc}, \mathrm{Ti}, \mathrm{V}$, $\mathrm{Cr}, \mathrm{Mn}, \mathrm{Co}, \mathrm{Ni}$ and $\mathrm{Zn}$ are measured. Among the neutroncapture elements we have estimated the abundances of elements Sr, Y, Zr, Ba, La, Ce, Pr, Nd, Sm, and Eu. For elements Sc, V, Mn, Ba, La and Eu, we have also used spec- 
Table 5: Comparison of our estimated stellar parameters with literature values

\begin{tabular}{lcccccc}
\hline Star & $\begin{array}{c}\mathrm{T}_{\text {eff }} \\
(\mathrm{K})\end{array}$ & $\begin{array}{c}\log \mathrm{g} \\
(\mathrm{cgs})\end{array}$ & $\begin{array}{c}\zeta \\
\left(\mathrm{km} \mathrm{s}^{-1}\right)\end{array}$ & {$[\mathrm{Fe} \mathrm{I} / \mathrm{H}]$} & {$[\mathrm{Fe} \mathrm{II} / \mathrm{H}]$} & Ref. \\
\hline HE 0308-1612 & 4600 & 1.70 & 1.42 & -0.72 & -0.73 & 1 \\
CD-28 1082 & 5200 & 1.90 & 1.42 & -2.46 & -2.44 & 1 \\
HD 29370 & 4970 & 2.40 & 1.92 & -0.15 & -0.13 & 1 \\
& 4800 & 2.10 & 1.60 & -0.25 & -0.28 & 2 \\
HD 30443 & 4040 & 2.05 & 2.70 & -1.68 & -1.69 & 1 \\
CD-38 2151 & 4600 & 0.90 & 2.30 & -2.03 & -2.03 & 1 \\
& 4700 & 1.5 & 3.00 & $-1.50^{a}$ & & 3 \\
HD 50264 & 5900 & 4.60 & 0.62 & -0.14 & -0.11 & 1 \\
& 5800 & 4.20 & 1.00 & -0.34 & -0.37 & 4 \\
HD 87080 & 5600 & 3.74 & 1.10 & -0.47 & -0.49 & 1 \\
& 5600 & 4.0 & 1.2 & -0.51 & -0.49 & 4 \\
HD 87853 & 5460 & 3.70 & 1.00 & -0.49 & -0.44 & 5 \\
HD 123701 & 6250 & 2.50 & 1.63 & -0.72 & -0.73 & 1 \\
& 5340 & 3.5 & 1.44 & 0.05 & 0.07 & 1 \\
HD 176021 & 5000 & 2.5 & 1.60 & -0.44 & -0.46 & 2 \\
& 5900 & 3.95 & 1.02 & -0.62 & -0.65 & 1 \\
HD 188985 & 6000 & 4.00 & 2.00 & -0.32 & -0.50 & 6 \\
& 6250 & 4.30 & 0.60 & 0.01 & 0.01 & 1 \\
& 6090 & 4.30 & 1.10 & -0.25 & -0.30 & 5 \\
HD 202020 & 5960 & 3.78 & 1.40 & -0.51 & -0.32 & 7 \\
& 5960 & 4.38 & 0.86 & 0.04 & 0.08 & 1 \\
& 5600 & 4.00 & 1.20 & -0.14 & -0.07 & 8 \\
\hline
\end{tabular}

Note. References: 1. Our work, 2. de Castro et al. (2016), 3. Vanture (1992), 4. Pereira \& Junqueira (2003), 5. Allen \& Barbuy (2006a), 6. Sneden \& Bond (1976), 7. North et al. (1994a,b) 8. Luck \& Bond (1991)

${ }^{a}$ Vanture (1992) value refers to $[\mathrm{Fe} / \mathrm{H}]$.

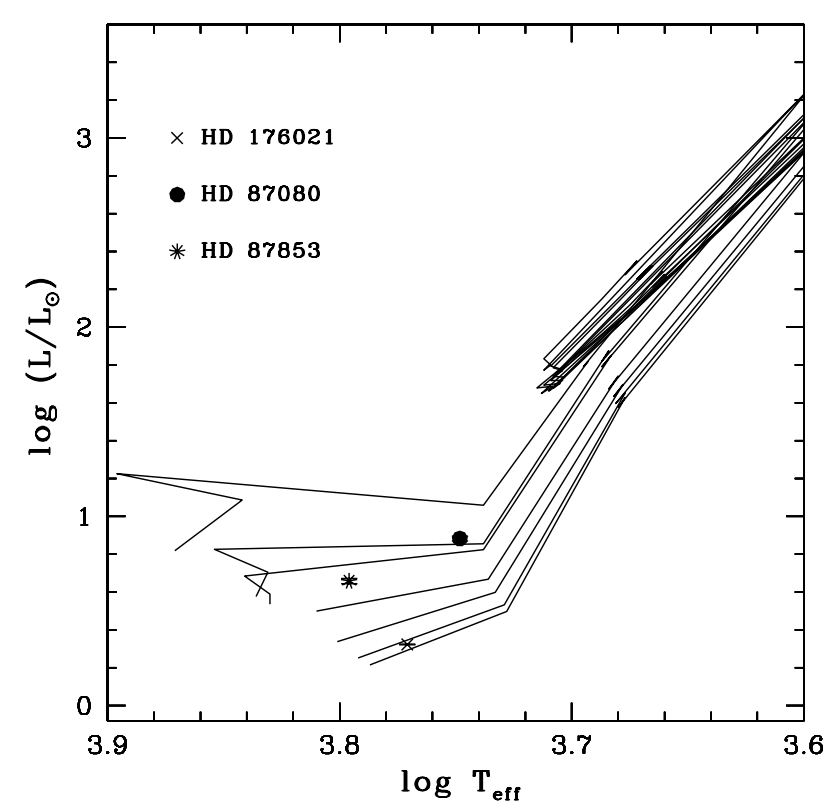

Figure 5. The isochrone tracks for $\log$ (age) 10.05, 10.0, 9.9, 9.8, $9.55,9.5$, and 9.3 from bottom to top.

trum synthesis calculation taking into account their hyperfine structures.

The abundance results are presented in Tables A1 through A4 and the lines used for the abundance determination are presented in Tables A5-A8.

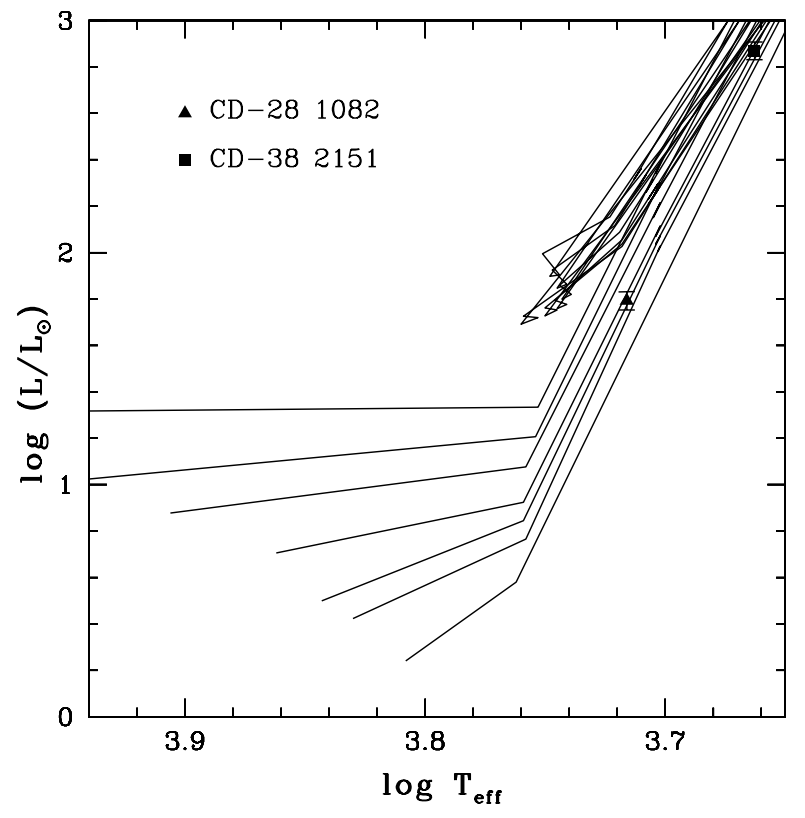

Figure 6. The isochrone tracks for CD-28 1082 and $C D-38$ 2151 corresponding to $\log$ (age) $10.2,10,9.9,9.8,9.6,9.4$ and 9.25 from bottom to top.

\subsection{Carbon, Nitrogen, Oxygen}

Oxygen abundance is determined using spectrum synthesis calculation of the oxygen forbidden lines [OI] $6300.3 \AA$ and $6363.8 \AA$ lines for the objects CD-38 2151, HD 30443, HD 123701, HD 202020. Some examples of spectrum synthesis fits are shown in Fig. 11. In case of the objects 


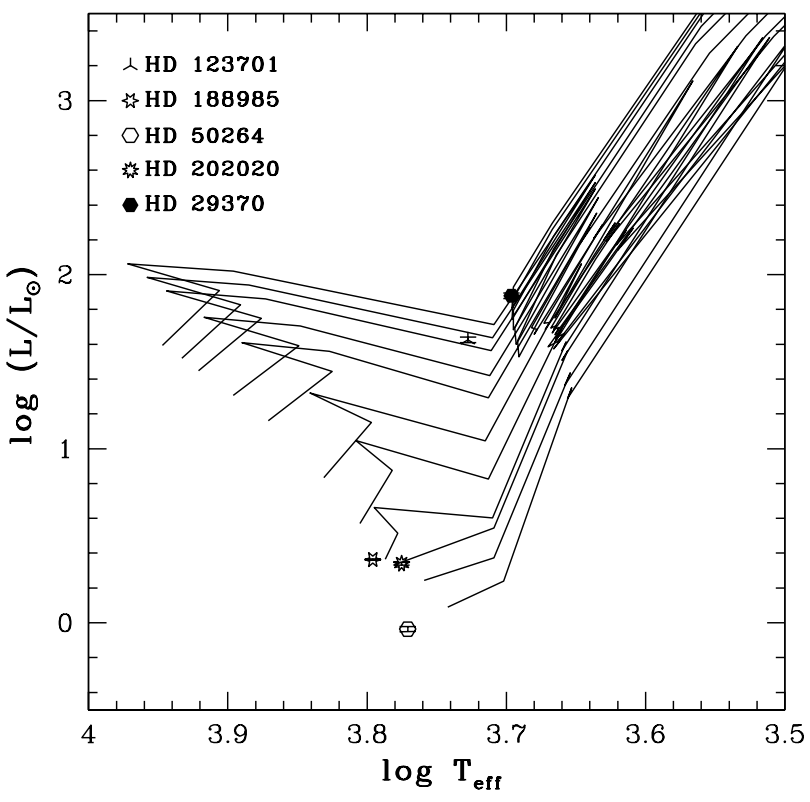

Figure 7. The isochrone tracks for $\log$ (age) 10.20, 10.0, 9.70, $9.60,9.40,9.20,9.00,8.90,8.80,8.75$, and 8.70 are shown from bottom to top.

HD 188985, HD 50264, HD 87080 and HD 87853 where these lines could not be measured we have determined the oxygen abundance using spectrum synthesis calculation of the triplet lines around $7774 \AA$ region (Fig. 12). These lines are influenced by non-LTE effects. Non-LTE corrections are estimated using the equation given in Bensby, Feltzing \& Lundstrom (2004). The NLTE effects on oxygen triplet decreases in objects, such as $\mathrm{K}$ giants with higher gravities, and it vanishes for lines with equivalent widths below 45 $\mathrm{m} \AA$ (Eriksson \& Toft 1979). As in most of the stars the O I triplet lines are found to be stronger with equivalent width slightly larger than $45 \mathrm{~m} \AA$, and hence we have applied nonLTE corrections to the estimated abundances. Oxygen is found to be near solar in HD 188985, whereas it is underabundant in HD 202020 with $[\mathrm{O} / \mathrm{Fe}] \sim-0.33$. For HD 50264 and HD 87080 the estimated $[\mathrm{O} / \mathrm{Fe}] \sim 0.37$. Oxygen is moderately enhanced in HD 87853, HD 123701 and CD-38 2151. In the rest of the objects the oxygen lines are found to be blended and not usable for abundance estimates.

Abundance of carbon is determined from spectrum synthesis calculation of the wavelength region 5162 to $5176 \AA$, including the $\mathrm{C}_{2}$ bandhead at $5165 \AA$. In this wavelength region there are no molecular features due to $\mathrm{MgH}, \mathrm{CN}$ bands. Atomic analysis of warm $\mathrm{K}$ and $\mathrm{M}$ dwarfs, shows that the molecular absorption is not strongly pronounced in spectra of stars at temperatures $\mathrm{T}_{\text {eff }}>3500 \mathrm{~K}$ (Woolf and Wallerstein 2005). The molecular lines used for the synthesis is taken from Kurucz database. Spectrum synthesis fits for a few programme stars are shown in Fig. 13.

CD-28 1082, HD 30443 and CD-38 2151 show carbon enhancement with $[\mathrm{C} / \mathrm{Fe}]>1$ while $\mathrm{HE} 0308-1612$ and HD 176021 are moderately enhanced with $[\mathrm{C} / \mathrm{Fe}] \sim 0.78$ and 0.52 , respectively. Abundance of carbon could not be estimated for HD 87853. In the remaining stars, $[\mathrm{C} / \mathrm{Fe}] \leq 0.23$ or near solar.
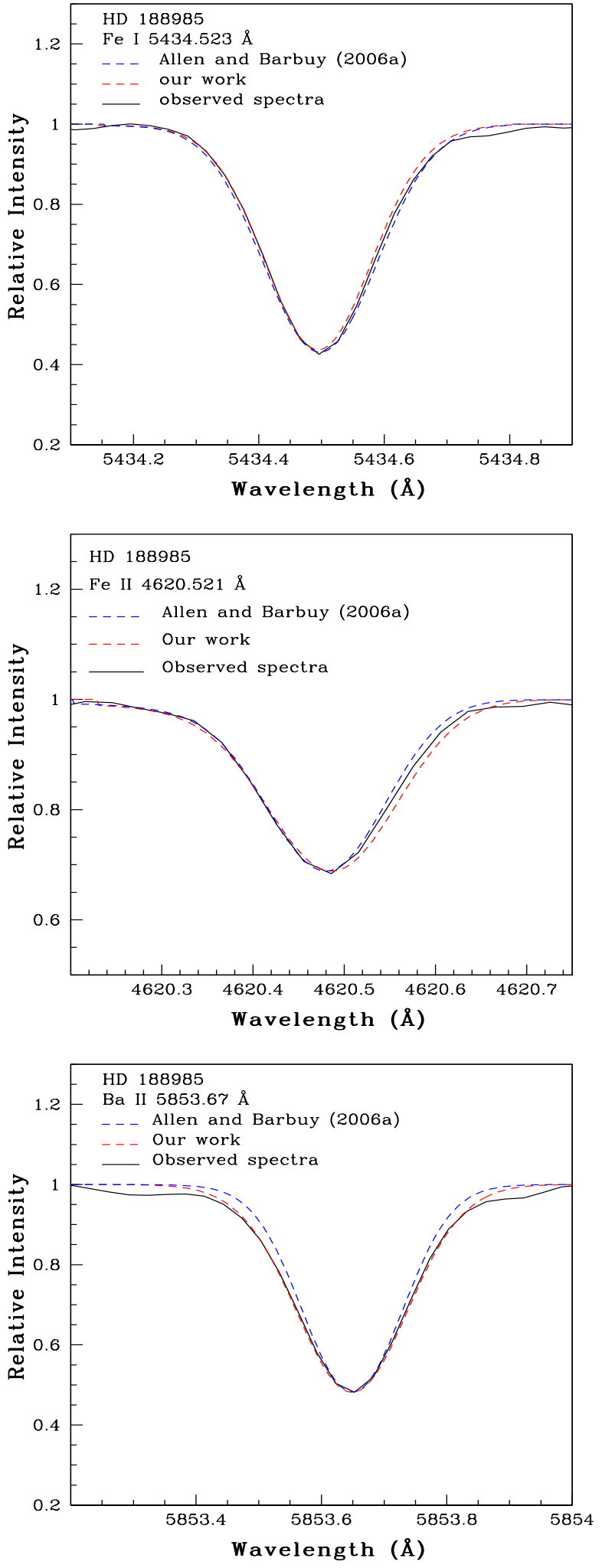

Figure 8. Synthesis of Fe I $5434.523 \AA$ (Top panel), Fe II $4620.521 \AA$ (Middle panel), and Ba II 5853.67 Å (Bottom panel). The best fits obtained with our atmospheric parameters and those of Allen \& Barbuy (2006a) are shown. 


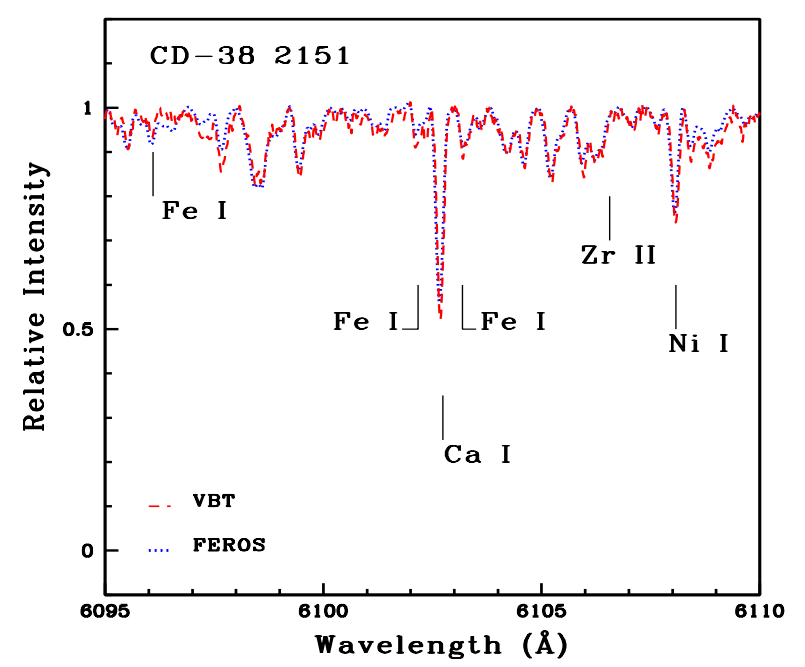

Figure 9. A comparison of the spectra of CD-38 2151 obtained with VBT with the FEROS spectrum shows a good match.

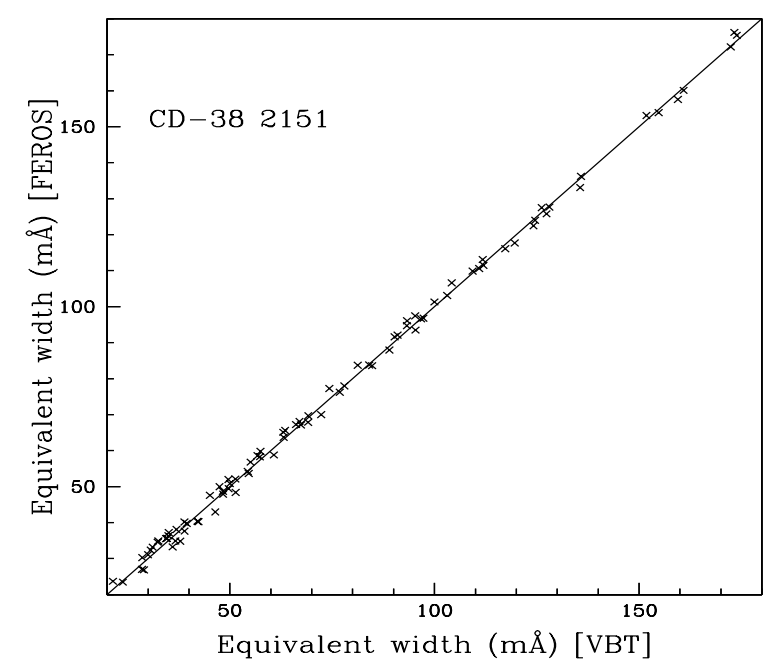

Figure 10. A comparison of the equivalent widths measured on the VBT spectrum of CD-38 2151 compared with those measured on the FEROS spectrum.

The carbon isotopic ratio ${ }^{12} \mathrm{C} /{ }^{13} \mathrm{C}$ is determined from the spectrum synthesis calculation of the $\mathrm{CN}$ band at 8005 $\AA$ (Fig. 14). We could estimate ${ }^{12} \mathrm{C} /{ }^{13} \mathrm{C}$ ratios for six objects HE 0308-1612, CD-28 1082, HD 29370, HD 30443, CD-38 2151 and HD 123701; the values are 15.6, 16, 7.4, 9.3, 11.2 and 10, respectively. In the rest of the stars this band is found to be either too weak or absent. While in CD-38 2151, HD 30443 and HD 202020, C/O is found to be $>1$ as normally seen in CH stars, HD 50264, HD 87080, HD 123701 and HD 188985 show $\mathrm{C} / \mathrm{O}<1$.

Nitrogen is estimated using the spectrum synthesis calculation of the $\mathrm{CN}$ band at $4215 \AA$ (Fig. 15). Nitrogen is enhanced in CD-28 1082 and CD-38 2151 with [N/Fe] 2.73 and 1.40 , respectively. The rest of the stars show moderate enhancement in nitrogen relative to iron. HE 0308-1612

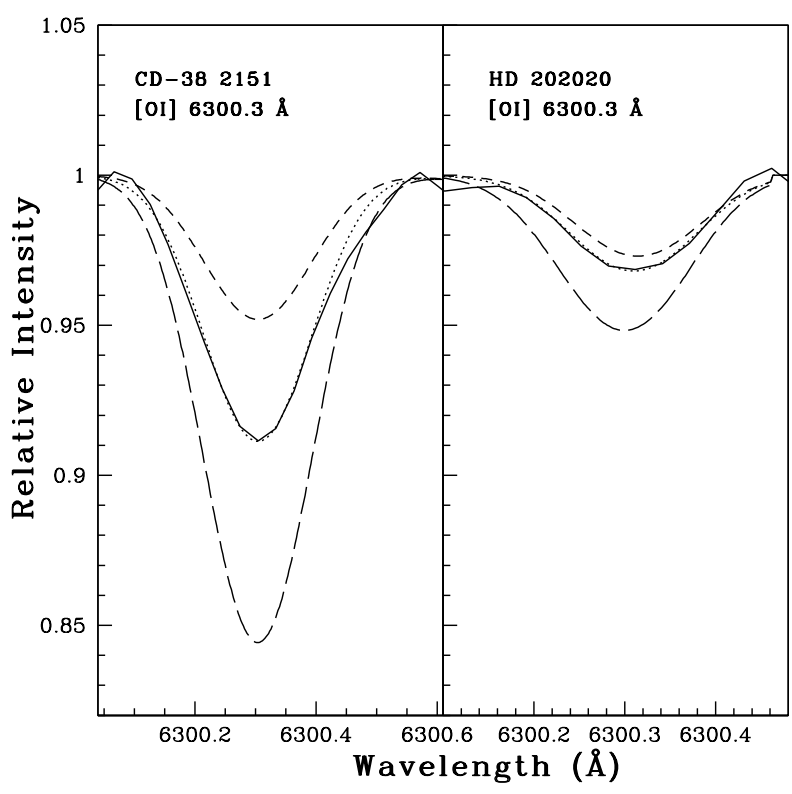

Figure 11. Synthesis of [OI] line around $6300 \AA$ A. Dotted line represents synthesized spectra and the solid line indicates the observed spectra. Short dashed line represents the synthetic spectra corresponding to $\Delta[\mathrm{O} / \mathrm{Fe}]=-0.3$ and long dashed line is corresponding to $\Delta[\mathrm{O} / \mathrm{Fe}]=+0.3$

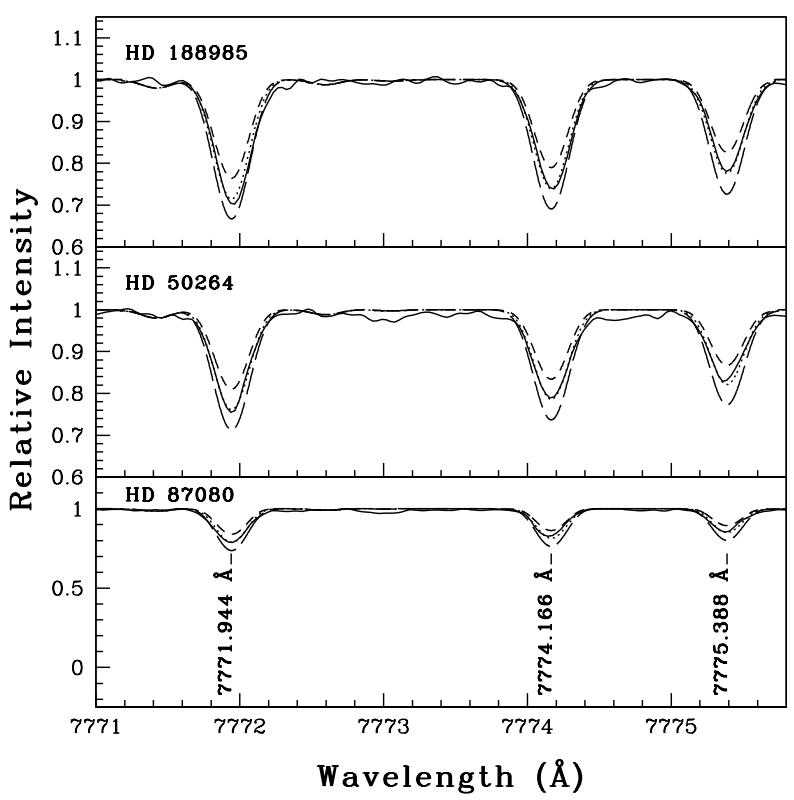

Figure 12. Synthesis of O I triplet. Dotted line represents synthesized spectra and the solid line indicates the observed spectra. Short dashed line represents the synthetic spectra corresponding to $\Delta[\mathrm{O} / \mathrm{Fe}]=-0.3$ and long dashed line is corresponding to $\Delta[\mathrm{O} / \mathrm{Fe}]=+0.3$ 


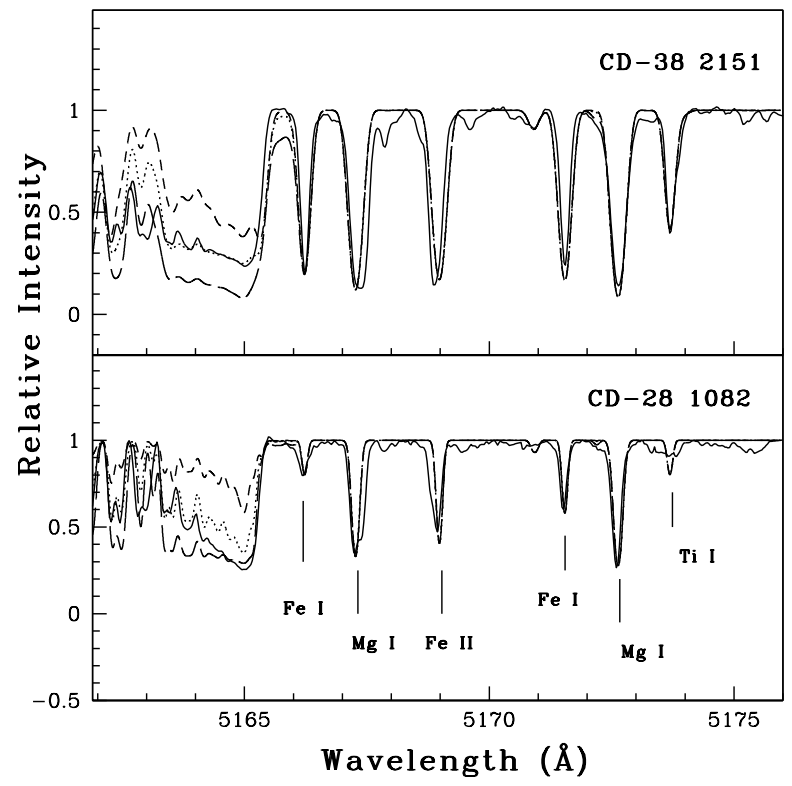

Figure 13. Synthesis of $\mathrm{C}_{2}$ band around $5165 \AA$. Dotted line represents synthesized spectra and the solid line indicates the observed spectra. Short dashed line represents the synthetic spectra corresponding to $\Delta[\mathrm{C} / \mathrm{Fe}]=-0.3$ and long dashed line is corresponding to $\Delta[\mathrm{C} / \mathrm{Fe}]=+0.3$

and HD 29370 show near solar values. Abundance of Nitrogen could not be estimated for HD 87853 .

\subsection{Na, Mg, Si, Ca, Sc, Ti, V}

The estimated $\mathrm{Na}$ abundances lie in the range 0.07-0.78. While five objects, HD 29370, HD 87080, HD 123701, HD 176021 and HD 202020 show solar values. CD -28 1082 shows an enhancement with $[\mathrm{Na} / \mathrm{Fe}] \sim 0.78$. Such an enhancement is not uncommon for stars at the metallicity of this star (Aoki et al. 2007). The lines used for abundance estimates are presented in Tables A7 and A8. We could not estimate the abundance of sodium in CD-38 2151, HD 50264 and HD 188985 as no suitable lines were found for abundance estimates.

Abundance of $\mathrm{Mg}$ is determined using lines $\mathrm{Mg} \mathrm{I}$ 4571.10, 4730.03, 5172.684, 5528.405, and 5711.09 $\AA$ whenever possible using spectrum synthesis calculations. For all the stars, the lines used for abundance analysis have equivalent widths in the range $20-180 \mathrm{~m} \AA$. In the case of HE 03081612 since only two Mg lines, Mg I 5528.405, and $4702.99 \AA$ are found to be useful for abundance analysis, we have used both the lines although Mg I 5528.405 has equivalent width slightly larger than $180 \mathrm{~m} \AA$ (i.e., $190.6 \mathrm{~m} \AA$ ). In CD-28 $1082, \mathrm{Mg}$ is found to be enhanced with $[\mathrm{Mg} / \mathrm{Fe}] \sim 0.45$. In CD-38 2151, this value is slightly higher with $[\mathrm{Mg} / \mathrm{Fe}] \sim$ 0.63 . HD 87853 also shows a similar value with $[\mathrm{Mg} / \mathrm{Fe}] \sim$ 0.68 . A value of 0.38 is obtained for HD 176021. For the rest of the objects we have obtained near solar values (Tables A1 - A4).

Abundance of $\mathrm{Si}$ is determined using lines, Si I 6145.02, 6155.13 and $6237.32 \AA$ whenever possible. While HD 29370 and HD 87080 show a mild deficiency with $[\mathrm{Si} / \mathrm{Fe}]=-0.36$

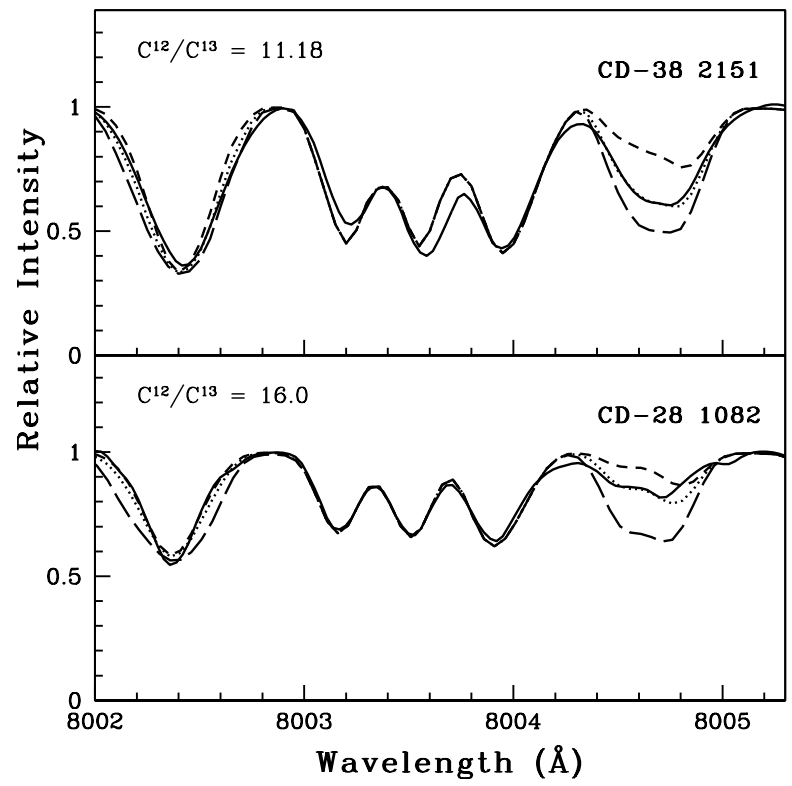

Figure 14. Synthesis of CN band around $8005 \AA$. Dotted line represents synthesized spectra and the solid line indicates the observed spectra and the synthetic spectra corresponding to ${ }^{12} \mathrm{C} /{ }^{13} \mathrm{C} \simeq 12$ (short dashed) and 1 (long dashed)

and -0.44 respectively. CD-38 2151 and HD 30443 give values of $[\mathrm{Si} / \mathrm{Fe}]=1.62$ and 0.82 respectively. In the rest of the objects abundance of Si could not be determined as no good lines were found for abundance determination.

Ca shows a mild enhancement of $[\mathrm{Ca} / \mathrm{Fe}]=0.27,0.37$, 0.29 in CD-28 1082, HE 0308-1612, HD 87853. Ca is also moderately enhanced in HD 30443 and CD-38 2151 with values $\sim 0.51$ and 0.58 respectively. HD 176021 shows a near solar value, and for the remaining six objects the $[\mathrm{Ca} / \mathrm{Fe}]$ lies in the range from -0.25 to -0.08 .

Abundance of Sc determined using the equivalent width

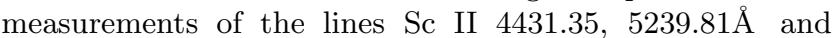
$5526.79 \AA$ give near solar values for $\mathrm{CD}-382151$ and HD 29370 with $[\mathrm{Sc} / \mathrm{Fe}] \sim 0.18$ and 0.19 respectively. Spectrum synthesis of Sc II $6245.63 \AA$ is used to estimate the abundance of $\mathrm{Sc}$ in $\mathrm{HE} 0308-1612$, that gives [Sc/Fe] $\sim 0.53$, and near solar values for HD 30443, HD 50264, HD 87080, HD 123701, HD 176021, and HD 202020. The objects HD 188985 and HD 87853 show mild deficiencies with $[\mathrm{Sc} / \mathrm{Fe}] \sim-0.21$ and -0.39 respectively. The hyperfine structure of Sc is taken from Prochaska and McWilliam (2000). Abundance of Sc could not be measured in CD-28 1082 as no good lines were found for abundance determination.

Abundance of $\mathrm{Ti}$ is measured using the equivalent width measurements of several Ti I lines (Tables A7, A8). [Ti/Fe] covers a range -0.28 to 0.32 , in these objects except for $\mathrm{CD}-382151$ where $[\mathrm{Ti} / \mathrm{Fe}]$ is 0.71 . The estimates of [Ti $\mathrm{II} / \mathrm{Fe}]$ covers a range -0.28 to +0.42 . We could not estimate abundance of $\mathrm{Ti}$ for $\mathrm{CD}-281082$ as no good lines were found.

The abundance of $\mathrm{V}$ measured from V I 6119.52, and $6251.83 \AA$ lines give a very high value with $[\mathrm{V} / \mathrm{Fe}] \sim 1.16$ for CD-38 2151. The reason for such a high value is not 


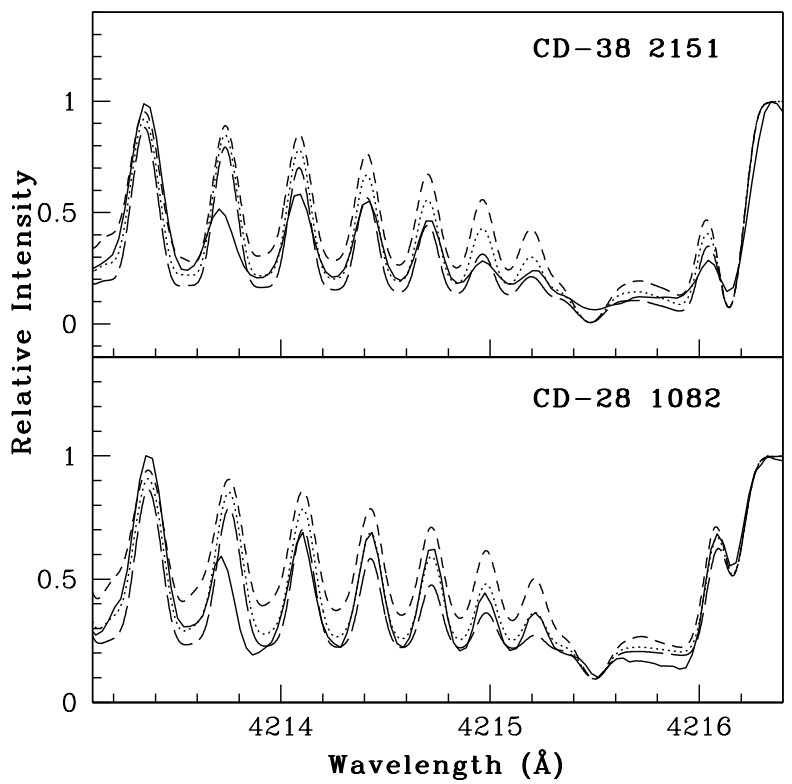

Figure 15. Synthesis of CN band around $4215 \AA$. Dotted line represents synthesized spectra and the solid line indicates the observed spectra. Short dashed line represents the synthetic spectra corresponding to $\Delta[\mathrm{N} / \mathrm{Fe}]=-0.3$ and long dashed line is corresponding to $\Delta[\mathrm{N} / \mathrm{Fe}]=+0.3$

understood. For HD 29370 and HD 123701 we get [V/Fe] values as 0.29 and 0.12 . The spectrum synthesis calculation of the lines V I 5727.048, 5727.652, and 5737.06 $\AA$ taking hyperfine splitting into considerations returns $[\mathrm{V} / \mathrm{Fe}]$ values $0.29,-0.64,-0.43$ and -0.18 for HE $0308-1612$, HD 30443, HD 176021 and HD 202020 respectively. Hyperfine structure is taken from Prochaska \& McWilliam (2000). The abundance of $\mathrm{V}$ could not be estimated for the remaining four objects.

\subsection{Cr, Co, Mn, Ni, Zn}

The abundance of $\mathrm{Cr}$ is derived using equivalent width measurements of several Cr I lines; [Cr I/Fe] ranges from -0.39 to 0.30 in these objects. We could also estimate abundance of Cr using Cr II lines in HD 29370, HD 50264, HD 87080, HD 87853 and HD 176021; [Cr II/Fe] ranges from -0.18 to 0.03 in these objects. Cromium abundance could not be estimated for CD-28 1082 and HD 30443.

The abundance of $\mathrm{Mn}$ is determined using the spectrum synthesis calculation of the Mn I 6013.51 A considering the hyperfine structure from Prochaska \& McWilliam (2000). CD-28 1082 shows a large enhancement of Mn with $[\mathrm{Mn} / \mathrm{Fe}] \sim 1.48$. HD 30443 shows near solar value. HD 29370 and HD 176021 give a mild enhancement with [Mn/Fe] values 0.32 and 0.17 respectively. Spectrum synthesis calculation of Mn I $4041.355 \AA$ gives a value -0.37 for HD 87853 . Abundance of $\mathrm{Mn}$ derived using equivalent width measurements of lines, Mn I 4739.087, 4766.418 and $4783.427 \AA$ is found to be $\sim 0.29$ with respect to iron for HE $0308-1612$. In the rest of the stars $[\mathrm{Mn} / \mathrm{Fe}]$ ranges from -0.38 to -0.2 .

Abundance of Co is measured using the Co I 4121.31, 4792.85, 4813.47, 5483.34 and $6632.43 \AA$ lines whenever possible. For CD-38 2151, HD 30443, HD 87853 and HD 123701, abundance of Co is found to be near solar. For HD 176021 and HD 188985 [Co/Fe] values are found to be mildly enhanced with $[\mathrm{Co} / \mathrm{Fe}] \sim 0.29$ and 0.33 respectively. For all other stars $[\mathrm{Co} / \mathrm{Fe}]$ ranges from -0.01 to -0.11 . The abundance of Co could not be estimated for HE 0308-1612 and CD-28 1082 as no good lines could be measured.

Abundance of $\mathrm{Ni}$ is measured using several Ni I lines (Tables A7 and A8). [Ni/Fe] ranges from 0.59 to -0.13 . The abundance of Ni could not be estimated for CD-28 1082 .

Abundance of $\mathrm{Zn}$ measured using only one line at $4810.53 \AA$ gives near solar values for HD 29370, HD 87080, HD 87853 and HD 123701. For stars HD 30443, HD 50264, HD 87853, HD 176021, HD 188985, and HD 202020 the $[\mathrm{Zn} / \mathrm{Fe}]$ values are found to lie in the range 0.21-0.40. HE 0308-1612 gives a value -0.19 . We could not estimate the abundance of $\mathrm{Zn}$ for CD-28 1082 and CD-38 2151 as no good lines are available in their spectra.

\subsection{Sr, Y, Zr}

Abundance of $\mathrm{Sr}$ is determined from spectrum synthesis calculation of Sr I $4607.33 \AA$. Strontium is enhanced in most of the stars with $[\mathrm{Sr} / \mathrm{Fe}]>1$. In HD 29370 and HD 30443, $\mathrm{Sr}$ is moderately enhanced with $[\mathrm{Sr} / \mathrm{Fe}] \sim 0.82$ and 0.74 respectively. A few examples of the spectrum synthesis fits for $\mathrm{Sr}$ is shown in Fig. 16. Abundance of Sr could not be estimated for HD 87853.

Abundance of $\mathrm{Y}$ is measured using several lines of $\mathrm{Y}$ II (Tables A7, A8). In HD 29370 and CD-38 2151 yttrium is enhanced with $[\mathrm{Y} / \mathrm{Fe}]$ values 0.87 and 0.57 respectively. In HD 87853 yttrium is underabundant with $[\mathrm{Y} / \mathrm{Fe}] \sim-0.48$. In all other stars yttrium is enhanced with $[\mathrm{Y} / \mathrm{Fe}]>1.0$; with highest enhancement seen in CD-28 1082 with [Y/Fe] $\sim 1.61$.

Abundance of $\mathrm{Zr}$ is determined using the measured equivalent width of $\mathrm{Zr}$ II $4210.631 \AA$. $\mathrm{Zr}$ is found to be enhanced in HD 29370, CD-38 2151, HD 123701 and HD 202020 with $[\mathrm{Zr} / \mathrm{Fe}]$ values $1.64,2.0,0.83$ and 0.48 respectively. Spectrum synthesis calculation of Zr I $6134.59 \AA$ gives $[\mathrm{Zr} / \mathrm{Fe}] \sim 1.60$ for HD 30443. In the rest of the objects the abundance of $\mathrm{Zr}$ could not be estimated as no good lines of $\mathrm{Zr}$ was found.

\subsection{Ba, La, Ce, Pr, Nd, Sm, Eu}

The abundance of barium is determined from the spectrum synthesis calculation of the Ba II $5853.668 \AA$ (Fig. 17) considering the hyperfine structure from McWilliam (1998). For HD 30443 we have used spectrum synthesis calculation of Ba II $6141.713 \AA$. Except for HD 87853 where barium is underabundant, all of our programme stars show Ba enhancement with $[\mathrm{Ba} / \mathrm{Fe}]$ in the range $0.8-2.1$.

Abundance of La is also determined from spectral synthesis calculation of La II $4921.778 \AA$ with the hyperfine structure taken from Jonsell et al. (2006). La is overabundant in most of the stars with $[\mathrm{La} / \mathrm{Fe}]$ ranging from 0.75 to 1.75. Abundance of La could not be estimated for HD 30443 and HD 87853.

The abundance of cerium is estimated using the equivalent width measurements of several Ce II lines (Tables A7, 

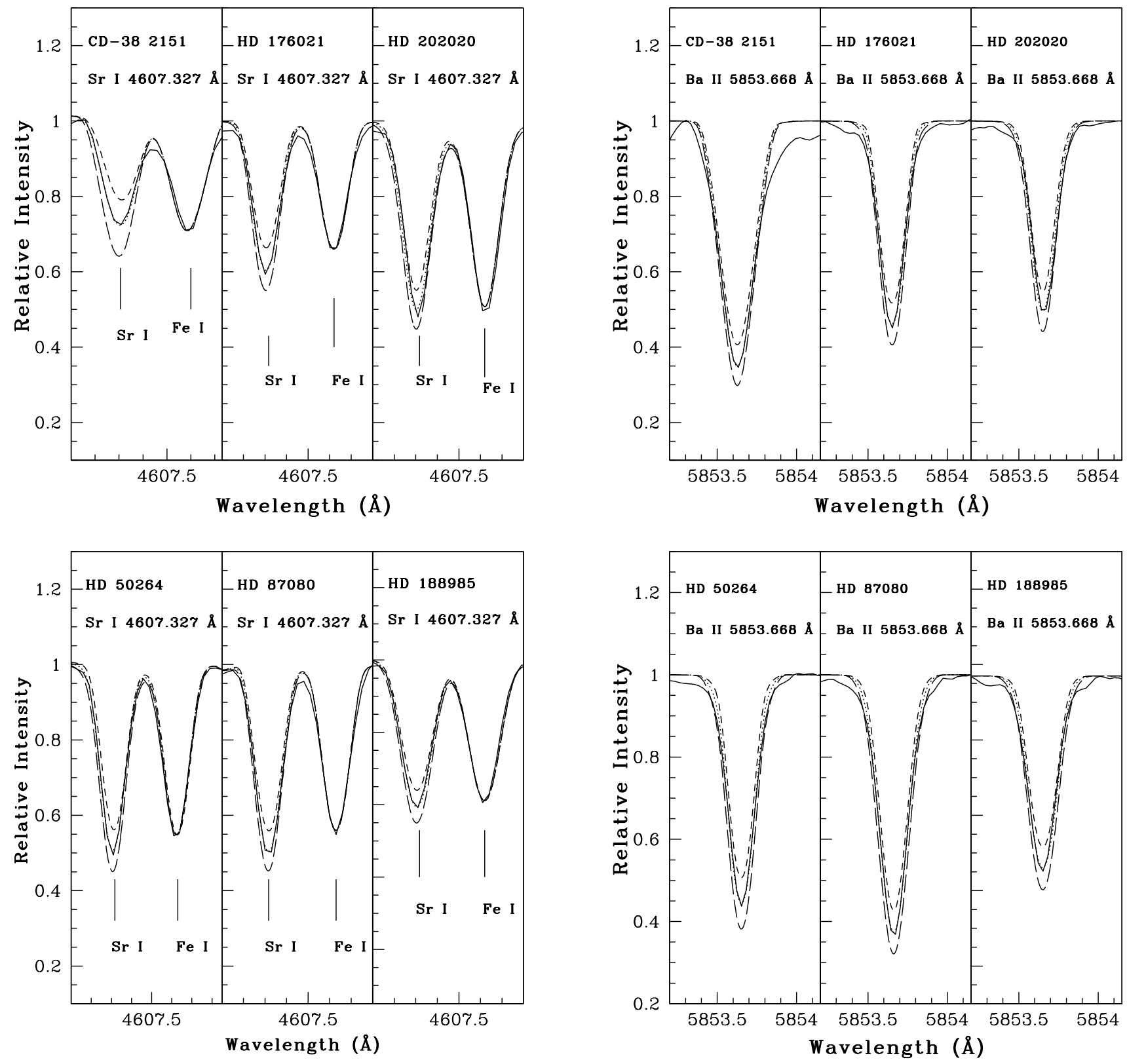

Figure 16. Synthesis of Sr I around $4607 \AA$. Dotted line represents synthesized spectra and the solid line indicates the observed spectra. Short dashed line represents the synthetic spectra corresponding to $\Delta[\mathrm{Sr} \mathrm{I} / \mathrm{Fe}]=-0.3$ and long dashed line is corresponding to $\Delta[\mathrm{Sr} \mathrm{I} / \mathrm{Fe}]=+0.3$

A8). The $[\mathrm{Ce} / \mathrm{Fe}]$ values are found to lie in the $0.8-1.97$. We could not estimate Ce abundance for HD 87853.

The abundance of $\mathrm{Pr}$ is measured using the equivalent width measurements of Pr II lines (A7, A8) that show enhancement in HE 0308-1612, CD-28 1082, HD 30443, CD-38 2151, HD 123701, and HD 87080 with $[\mathrm{Pr} / \mathrm{Fe}]$ in the range 1.52-2.67. Abundance of Pr could not be measured for HD 29370, HD 50264, HD 87853, HD 176021, HD 188985 and HD 202020.

Abundance of $\mathrm{Nd}$ is measured using equivalent width measurements of several Nd lines (Tables A7, A8). Neodim-

Figure 17. Synthesis of Ba II around 5835 A. Dotted line represents synthesized spectra and the solid line indicates the observed spectra. Short dashed line represents the synthetic spectra corresponding to $\Delta[\mathrm{Ba} \mathrm{II} / \mathrm{Fe}]=-0.3$ and long dashed line is corresponding to $\Delta[\mathrm{Ba} I \mathrm{II} / \mathrm{Fe}]=+0.3$

ium is found to be enhanced with $[\mathrm{Nd} / \mathrm{Fe}]>1$, in all the stars except for HD 87853 and HD 202020.

The abundance of $\mathrm{Sm}$ is measured using equivalent width measurements of a maximum of five samarium lines. $\mathrm{Sm}$ is enhanced in CD-28 1082 and HD 30443 with [Sm/Fe] $\sim 2.29$ and 2.24 respectively. HE $0308-1612$, HD 29370, CD-38 2151, HD 87080, HD 123701 and HD 176021 show enhancement with $[\mathrm{Sm} / \mathrm{Fe}]$ in the range 1.41-1.68. Abundance of Sm could not be estimated for HD 50264, HD 87853, HD 188985, and HD 202020 as no good lines of Sm were available for abundance estimation. 
Eu abundance is determined for six objects using spectrum synthesis calculation of lines Eu II $4129.71 \AA$, 6437.63 $\AA$, and $6645.11 \AA$ considering hyperfine structure from Worley et al. (2013). CD-28 1082 shows enhancement with $[\mathrm{Eu} / \mathrm{Fe}] \sim 2.07$ measured using only one line Eu II 6437.63 A. In HD 123701, this line shows a marginal enhancement with $[\mathrm{Eu} / \mathrm{Fe}]=0.15$. For HE 0308-1612 and HD 87080 we have used the $\mathrm{Eu}$ II $6645.11 \AA$ that gives $[\mathrm{Eu} / \mathrm{Fe}] \sim 0.36$. Abundance of Eu estimated using the line Eu II 4129.71 $\AA$ gives [Eu/Fe] values $0.38,0.10$ and 0.07 for HD 176021, 188985 and HD 202020. Abundance of Eu measured using equivalent width measurement of $\mathrm{Eu}$ II $4205.042 \AA$ gives $[\mathrm{Eu} / \mathrm{Fe}] \sim 0.34$ for HD 87853. Abundance of $\mathrm{Eu}$ could not be estimated for HD 29370, HD 30443, CD-38 2151, and HD 50264 as no good lines are available for abundance estimation.

We have estimated [ls/Fe], [hs/Fe] and [hs/ls], where ls stands for the light s-process elements (Sr, Y, Zr) and hs stands for the heavy s-process ( $\mathrm{Ba}, \mathrm{La}, \mathrm{Ce}, \mathrm{Nd}, \mathrm{Sm}$ ) elements; these values are presented in Table 6 .

\section{KINEMATIC ANALYSIS}

Space velocity of the programme stars are calculated using the method described in Bensby, Feltzing \& Lundstrom (2003). The space velocity with respect to the Local Standard of Rest (LSR) is given by

$$
(U, V, W)_{L S R}=(U, V, W)+(U, V, W) \odot \mathrm{km} / \mathrm{s} .
$$

where, $(U, V, W)_{\odot}=(11.1,12.2,7.3) \mathrm{km} / \mathrm{s}$ (Schönrich et al., 2010) is the solar motion with respect to LSR, and

$$
\left[\begin{array}{l}
U \\
V \\
W
\end{array}\right]=B\left[\begin{array}{l}
k \mu_{\alpha} / \pi \\
k \mu_{\delta} / \pi \\
\rho
\end{array}\right]
$$

where $\mathrm{B}=\mathrm{T} . \mathrm{A}, \mathrm{k}=4.74057 \mathrm{~km} \mathrm{~s}^{-1}, \mu_{\alpha}$ is the proper motion in right ascension, in $\operatorname{arcsec} \mathrm{yr}^{-1}, \mu_{\delta}$ is the proper motion in declination, in arcsec $\mathrm{yr}^{-1}, \rho$ is the radial velocity in $\mathrm{kms}^{-1}$ and $\pi$ is the parallax in arcsec.

The transformation matrix connecting Galactic coordinates and equatorial coordinates is:

$T=\left[\begin{array}{lll}-0.0548756 & -0.8734371 & -0.4838350 \\ +0.4941094 & -0.4448296 & +0.7469822 \\ -0.8676661 & -0.1980764 & +0.4559838\end{array}\right]$

Coordinate matrix A can be defined as:

$A=\left[\begin{array}{ccc}-\sin \alpha & -\cos \alpha \sin \delta & +\cos \alpha \cos \delta \\ +\cos \alpha & -\sin \alpha \sin \delta & \sin \alpha \cos \delta \\ 0 & +\cos \delta & \sin \delta\end{array}\right]$

Where $\alpha$ is the right ascension and $\delta$ is the declination in degrees. We are using a right-handed coordinate system for $\mathrm{U}, \mathrm{V}$ and $\mathrm{W}$. Hence they are positive in the directions of the Galactic center, Galactic rotation, and the North Galactic Pole (NGP) respectively (Johnson \& Soderblom (1987)).

The estimates for proper motion are taken from SIMBAD. Distances are measured taking parallax values from SIMBAD and Gaia (Gaia Collaboration 2016, Gaia Collaboration et al. 2018b) whenever available. We have used the radial velocity estimates and the corresponding error estimates as measured by us.

The total spatial velocity of a star is given by, $V_{s p a}^{2}=U_{L S R}^{2}+V_{L S R}^{2}+W_{L S R}^{2}$

The probability for a star's membership into the thin disk, the thick disk or the halo population are also calculated following the procedures of Reddy, Lambert \& Priesto (2006), Bensby et al. (2003, 2004), Mishenina et al. (2004), and presented in Table 7, along with the estimated components of spatial velocity and the total spatial velocity. The selection of the thick or thin disk stars and halo stars are based on the assumption that the Galactic space velocities of these stars have Gaussian distributions.

$f(U, V, W)=K \times \exp \left[-\frac{U_{L S R}^{2}}{2 \sigma_{U}^{2}}-\frac{\left(V_{L S R}-V_{a s y}\right)^{2}}{2 \sigma_{V}^{2}}-\frac{W_{L S R}^{2}}{2 \sigma_{W}^{2}}\right]$

where

$K=\frac{1}{(2 \pi)^{\frac{3}{2}} \sigma_{U} \sigma_{V} \sigma_{W}}$

The values of the characteristic velocity dispersion $\sigma_{U}$, $\sigma_{V}$ and $\sigma_{W}$ and the asymmetric drift $V_{a s y}$ are adopted from Reddy et al. (2006).

\section{DISCUSSION}

In the following, we discuss the abundance patterns and other properties of the individual stars.

HE 0308-1612 - This object is listed among the 403 faint high latitude carbon stars of Hamburg/ESO survey(HES; Christlieb et al. 2001). From low-resolution spectroscopic analysis Goswami (2005) confirmed this object to be a potential $\mathrm{CH}$ star. In this work we have conducted a detailed chemical analysis based on a high resolution spectrum. The object is found to be mildly metal-poor with a metallicity of -0.73 . Further, with its estimated $[\mathrm{C} / \mathrm{Fe}] \sim 0.78$, $[\mathrm{Ba} / \mathrm{Fe}] \sim 1.63$ and $[\mathrm{Ba} / \mathrm{Eu}] \sim 1.28$, the star may be placed in the group of CEMP-s stars. As the oxygen abundance could not be estimated, this object could not be classified on the basis of $\mathrm{C} / \mathrm{O}$ ratio. The star has a low value of 15.6 for ${ }^{12} \mathrm{C} /{ }^{13} \mathrm{C}$ ratio.

CD-28 1082 - This star belongs to the CH star catalogue of Bartkevicius (1996). This work presents the first time abundance analysis for this object. The object is found to be a very metal-poor with a metallicity of -2.45 . Carbon is highly enhanced with $[\mathrm{C} / \mathrm{Fe}] \sim 2.19$. Barium as well as europium are also found to be enhanced relative to iron. The star satisfies the classification criteria for CEMP-r/s stars $([\mathrm{C} / \mathrm{Fe}]>1,[\mathrm{Ba} / \mathrm{Fe}]>1$ and $0.0 \leq[\mathrm{Ba} / \mathrm{Eu}] \leq 0.50)$ of Beers \& Christlieb (2005)).

A comparison of the the abundance ratios of light and heavy elements in CD-28 1082 with those observed in CEMP-r/s and $\mathrm{CH}$ stars from literature (Figs 18 and 19) shows a good match with the CEMP-r/s stars. Three CEMP-r/s stars from Aoki et al.(2007) exhibit very high Mg abundances (CS 29497-034 $([\mathrm{Mg} / \mathrm{Fe}]=1.31)$, CS 29528-028 $([\mathrm{Mg} / \mathrm{Fe}]=1.69)$, and $\mathrm{HE} 1447+0102([\mathrm{Mg} / \mathrm{Fe}]=1.43))$. The astrophysical processes that could produce such high enhancement of $\mathrm{Mg}$ are not clearly understood (Aoki et al. $2007)$. Our estimated value of $[\mathrm{Mg} / \mathrm{Fe}](=0.45)$ for $\mathrm{CD}-$ 281082 is close to that found by Magain (1989), who had obtained a mean value of $[\mathrm{Mg} / \mathrm{Fe}]=0.48 \pm 0.09$ dex from an analysis of 20 extremely metal-poor halo dwarfs in the metallicity range $-2.9<[\mathrm{Fe} / \mathrm{H}]<-1.4$. 
Table 6: Estimates of $[\mathrm{Fe} / \mathrm{H}],[\mathrm{ls} / \mathrm{Fe}],[\mathrm{hs} / \mathrm{Fe}],[\mathrm{hs} / \mathrm{ls}]$ and $\mathrm{C} / \mathrm{O}$

\begin{tabular}{lccccc}
\hline Star name & {$[\mathrm{Fe} / \mathrm{H}]$} & {$[\mathrm{ls} / \mathrm{Fe}]$} & {$[\mathrm{hs} / \mathrm{Fe}]$} & {$[\mathrm{hs} / \mathrm{ls}]$} & $\mathrm{C} / \mathrm{O}$ \\
\hline HE 0308-1612 & -0.73 & 1.11 & 1.59 & 0.48 & - \\
CD-28 1082 & -2.45 & 1.74 & 2.39 & 0.65 & - \\
HD 29370 & -0.14 & 1.14 & 1.28 & 0.14 & - \\
HD 30443 & -1.68 & 1.24 & 2.01 & 0.77 & 1.02 \\
CD-382151 & -2.03 & 1.24 & 1.28 & 0.04 & 2.95 \\
HD 50264 & -0.13 & 1.54 & 1.43 & -0.11 & 0.41 \\
HD 87080 & -0.48 & 1.48 & 1.64 & 0.16 & 0.29 \\
HD 87853 & -0.73 & -0.48 & 0.26 & 0.74 & - \\
HD 123701 & 0.06 & 1.19 & 1.62 & 0.43 & 0.15 \\
HD 176021 & -0.64 & 1.16 & 1.38 & 0.22 & - \\
HD 188985 & 0.01 & 1.60 & 1.40 & -0.20 & 0.89 \\
HD 202020 & 0.06 & 1.10 & 0.77 & -0.33 & 1.99 \\
\hline
\end{tabular}

Table 7: Spatial velocity and probability estimates for the programme stars

\begin{tabular}{|c|c|c|c|c|c|c|c|c|}
\hline Star name & $\begin{array}{c}\mathrm{U}_{L S R} \\
\left(\mathrm{kms}^{-1}\right)\end{array}$ & $\begin{array}{c}\mathrm{V}_{L S R} \\
\left(\mathrm{kms}^{-1}\right)\end{array}$ & $\begin{array}{c}\mathrm{W}_{L S R} \\
\left(\mathrm{kms}^{-1}\right)\end{array}$ & $\begin{array}{c}\mathrm{V}_{s p a} \\
\left(\mathrm{kms}^{-1}\right)\end{array}$ & $\mathrm{p}_{\text {thin }}$ & $\mathrm{p}_{\text {thick }}$ & $\mathrm{p}_{\text {halo }}$ & Population \\
\hline HE $0308-1612$ & $-180.36 \pm 17.11$ & $-90.63 \pm 10.0$ & $49.69 \pm 13.22$ & $207.87 \pm 15.98$ & 0.0 & 0.85 & 0.15 & Thick disk \\
\hline CD-28 1082 & $-3.95 \pm 1.17$ & $-132.96 \pm 6.99$ & $107.91 \pm 3.53$ & $171.28 \pm 3.23$ & 0.0 & 0.73 & 0.26 & Thick disk \\
\hline HD 29370 & $22.08 \pm 0.29$ & $18.85 \pm 0.19$ & $-1.95 \pm 0.24$ & $29.09 \pm 0.33$ & 0.99 & 0.01 & 0.0 & Thin disk \\
\hline HD 30443 & $-60.89 \pm 0.25$ & $-2.20 \pm 0.62$ & $11.72 \pm 0.30$ & $62.04 \pm 0.21$ & 0.98 & 0.02 & 0.0 & Thin disk \\
\hline CD-38 2151 & $-68.49 \pm 1.25$ & $-126.23 \pm 1.43$ & $6.64 \pm 3.01$ & $143.76 \pm 1.71$ & 0.02 & 0.95 & 0.03 & Thick disk \\
\hline HD 50264 & $-48.07 \pm 1.35$ & $-34.99 \pm 2.19$ & $11.67 \pm 0.68$ & $60.59 \pm 2.21$ & 0.97 & 0.03 & 0.0 & Thin disk \\
\hline HD 87080 & $-87.39 \pm 1.39$ & $16.13 \pm 0.14$ & $23.55 \pm 0.23$ & $91.94 \pm 1.24$ & 0.95 & 0.05 & 0.0 & Thin disk \\
\hline HD 87853 & $-43.92 \pm 0.96$ & $21.24 \pm 0.24$ & $-24.11 \pm 0.50$ & $54.42 \pm 0.90$ & 0.98 & 0.02 & 0.0 & Thin disk \\
\hline HD 123701 & $4.71 \pm 0.19$ & $30.51 \pm 0.24$ & $-0.88 \pm 0.19$ & $30.88 \pm 0.26$ & 0.99 & 0.01 & 0.00 & Thin disk \\
\hline HD 176021 & $75.65 \pm 0.41$ & $-113.05 \pm 0.33$ & $0.05 \pm 0.28$ & $136.03 \pm 0.05$ & 0.07 & 0.90 & 0.02 & Thick disk \\
\hline HD 188985 & $26.74 \pm 0.05$ & $17.18 \pm 0.03$ & $18.51 \pm 0.07$ & $36.78 \pm 0.09$ & 0.99 & 0.013 & 0.0 & Thin disk \\
\hline HD 202020 & $6.56 \pm 0.11$ & $10.98 \pm 0.11$ & $33.28 \pm 0.16$ & $35.65 \pm 0.21$ & 0.96 & 0.034 & 0.0 & Thin disk \\
\hline
\end{tabular}

Analysis of extremely metal-poor field giants in the metallicity range $-3.0<[\mathrm{Fe} / \mathrm{H}]<-1.3$ by Gratton \& Sneden (1988) suggested a mean value of $[\mathrm{Mg} / \mathrm{Fe}]=0.27$ dex with a star-to-star scatter of 0.14 dex. $[\mathrm{Mg} / \mathrm{Fe}]$ is found to be increasing with decreasing $[\mathrm{Fe} / \mathrm{H}]$. The analysis of Edvardsson et al. (1993) shows $[\mathrm{Mg} / \mathrm{Fe}] \sim 0.5$ dex at $[\mathrm{Fe} / \mathrm{H}]=$ -1.0 dex and attains near solar value at $[\mathrm{Fe} / \mathrm{H}]=0.0$ dex. They also report anomalously large value of $[\mathrm{Mg} / \mathrm{Fe}]$ for a few of the disk dwarfs in their sample. de Castro et al. (2016) analysed the variation of $[\mathrm{Mg} / \mathrm{Fe}]$ with $[\mathrm{Fe} / \mathrm{H}]$ for both barium and field giants that lie in a range from 0.0 to $0.5-0.6$ at $[\mathrm{Fe} / \mathrm{H}] \sim-1.5$. In the $\log \left({ }^{12} \mathrm{C} /{ }^{13} \mathrm{C}\right)$ versus $[\mathrm{C} / \mathrm{N}]$ plot of Masseron (2010), this object falls well within the region occupied by CEMP stars (Fig 20).

From kinematic analysis, CD-28 1082 is found to be a thick disk star with a probability of nearly 73 percent. The estimated spatial velocity $\left(\sim 180 \mathrm{~km} \mathrm{~s}^{-1}\right)$ is similar to that expected for halo objects (Chen, Nissen \& Zhao 2004). This object also satisfies the condition for $\mathrm{V}_{L S R}<-120 \mathrm{Km} \mathrm{s}^{-1}$ for it to be a halo star (Eggen 1997).

There exist several physical scenarios on the formation of CEMP-r/s stars (Jonsell et al. 2006, Bisterzo et al. 2011 and references there in). One of the possible scenarios is that, the CEMP-r/s stars are formed in a medium enriched in $\mathrm{r}$ process elements which is produced by a supernovae explosion in the past. Argast et al. (2004) studied the interstellar enrichment of r-process elements in the context of inhomegenous chemical evolution. Their studies show that core collapse supernovae are the dominant source of r-process elements compared to neutron star mergers. Calculations of

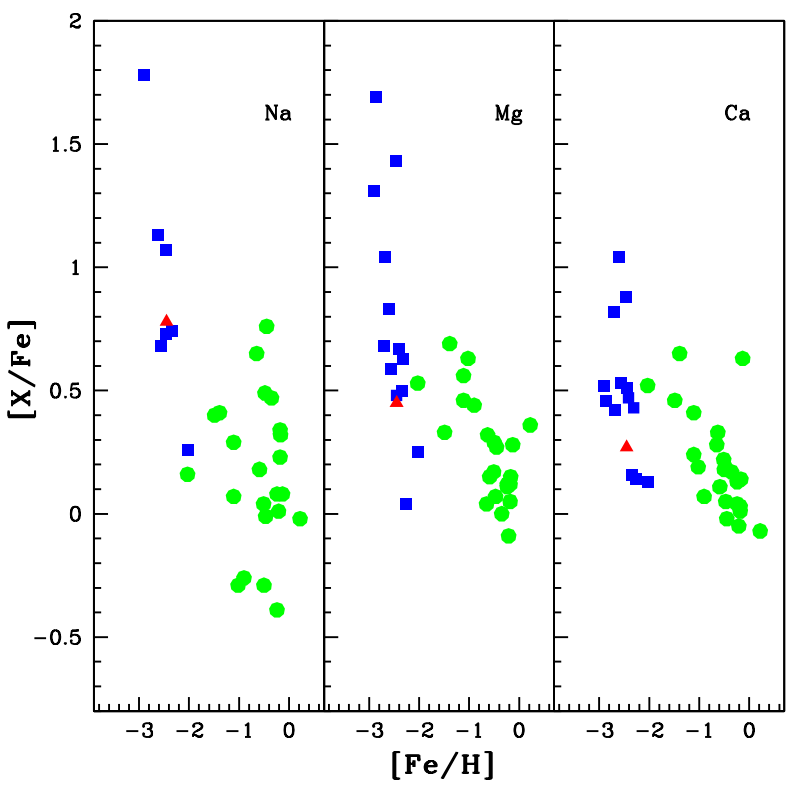

Figure 18. Comparison of abundances of light elements for CH stars (Sneden \& Bond 1976; Luck \& Bond 1991; Vanture 1992; Karinkuzi \& Goswami 2014, 2015) and CEMP-r/s stars (Masseron et al. 2010). Filled circles represent CH stars and the filled squares represent CEMP-r/s stars. The object CD-28 1082 is represented by a filled triangle. 

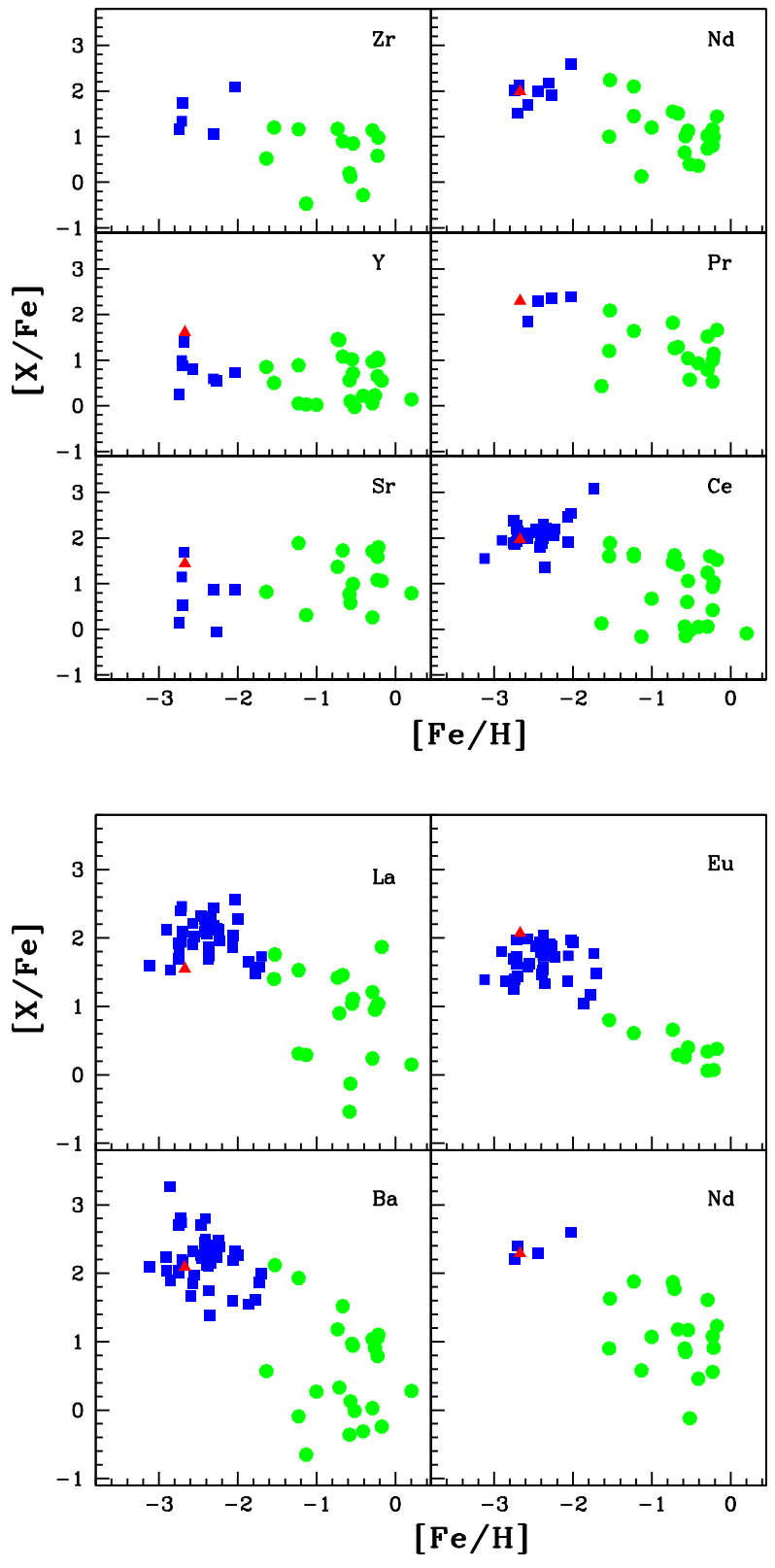

Figure 19. Comparison of abundances of heavy elements for CH stars (Sneden \& Bond 1976; Luck \& Bond 1991; Vanture 1992; Karinkuzi \& Goswami 2014, 2015) and CEMP-r/s stars (Masseron et al. (2010)). Filled circles represent $\mathrm{CH}$ stars and the filled squares represent CEMP-r/s stars. The object CD-28 1082 is represented by a filled triangle.

Rosswog et al. $(1999,2000)$ show that although the amount of r-process matter ejected in neutron star merger is much larger than that in supernovae collapse, the rate of neutron star merger in the galaxy is less than the Type II SNe (Tammann, Löffler \& Schröder 1994; Belczynski, Kalogera \& Bulik 2002 ). Hence it is likely that both of these sources might contribute to the r-process elements in the Galaxy (Qian \& Woosley 1996; Rosswog et al. 1999; Rosswog \& Davies 2002; Thielemann et al. 2002).

Bisterzo et al. (2011) suggest that the molecular cloud from which the binary system formed was previously polluted in r-process elements by some r-process sources, believed to have occurred during supernovae explosions and /or neutron star mergers (Thielemann et al. 2011, Wehmeyer et al. 2015). Argast et al. (2004) rule out neutron star merger as a dominant source of $r$-process since the expected $r$ process enrichment by neutron star merger is not found to be consistent with the observations at very low metallicity stars. However, Van de Voort et al. (2015) studied the abundance pattern of r-process elements using cosmological zoom-in simulations of a Milky Way-mass galaxy using various models for the rate and delay time of neutron star mergers and obtained results that are in contrast to those of Argast et al. (2004).

It was shown in Van de Voort et al. (2015) that the neutron star mergers can produce [r-process $/ \mathrm{Fe}]$ abundance ratios and scatter, consistent with the observations for stars with metallicity in the range $-2.0 \leq[\mathrm{Fe} / \mathrm{H}] \leq 0$. Despite some uncertainties in their model calculations, it was concluded that neutron star mergers can be an important source of the majority of the r-process elements. Investigation of the production sites of r-process elements in the Galaxy by Shen et al. (2015) based on the high resolution cosmological zoom-in simulation also concludes that neutron star mergers can be a dominant production site of r-process elements even at low metallicity. Haynes et al. (2018) studied the distribution of elemental abundance ratios using chemodynamical simulations including various neutron capture processes such as magneto-rotational supernovae, neutron star mergers, neutrino driven winds and electron capture supernovae. A comparison of their predicted trend between $[\mathrm{Eu} / \mathrm{Fe}]$ and $[\mathrm{Eu} / \mathrm{O}]$ as a function of metallicity with the observational data shows that neither electron capture supernovae nor neutrino driven winds could explain the observed Eu levels; however, neutron star mergers and magneto-rotational supernovae are able to justify the observed Eu abundances.

The possible explantion for the observed s-process enhancemet is attributed to a binary companion. Another scenario of CEMP-r/s stars formation is that it is born in a triple system (Cohen et al. 2003), in which one of the companions explodes as supernova. The tertiary star accretes the r-process rich material from this companion. The enhancement in carbon and s-process elements are explained by the accretion of materials from its secondary AGB companion. Cohen et al. (2003) also suggested another mechanism in which the CEMP-r/s stars are initially polluted by its binary companion which later becomes a white dwarf. At some point the reverse mass transfer can take place from the secondary star to the white dwarf. This may induce accretion induced collapse of the white dwarf if it is an O-Ne-Mg dwarf. The resultant neutrino driven wind is sufficient to contribute r-process elements on the surface of the secondary star. Yet another possible scenario is that the CEMP-r/s star is in a binary system and accretes material from its primary companion. Later the companion explodes as a type $1.5 \mathrm{su}-$ pernova (Zijlstra, 2004). Low metallicity stars with initial mass 3-4 M $\odot$ undergo this type of evolution (Zijlstra 2004). Abate, Stancliffe \& Liu (2016) have examined the possibility of different mechanisms of formation of CEMP-r/s stars but could not reproduce the observed frequency of CEMP-r/s stars by their simulations.

Several authors (Hansen et al. 2016; Hampel et al. 2016 
and references therein) have discussed the possibilities of iprocess in low metallicity AGB stars as the origin of CEMP$\mathrm{r} / \mathrm{s}$ stars. The neutron density associated with the i-process is sufficient to produce both the $\mathrm{s}$ and $\mathrm{r}$ process elements in AGB stars. Hampel et al. (2016) showed that the observed abundance pattern in many of the CEMP-r/s is consistent with the model calculations for i-process within the observational errors. It would be worthwhile to check in the future if the abundance pattern observed in CD-28 1082 can be explained on the basis of the i-process.

CD-38 2151 - This star belongs to the CH star catalogue of Bartkevicius (1996). Our abundance estimates are also characteristic of giant $\mathrm{CH}$ stars. The probability estimate shows this to be a thick disk object. With the lower bound, the spatial velocity estimate supports the inclusion of the star into the disk population while the upper bound in spatial velocity and the metallicity estimate shows this to be a halo object. It also satisfies the condition $\mathrm{V}_{L S R}<$ $-120 \mathrm{Kms}^{-1}$ for it to be a halo star (Eggen 1997).

Previous studies on this object include an abundance analysis by Vanture (1992) who had performed abundance estimates for six heavy elements based on spectra with typical resolution of $\lambda / \delta \lambda \sim 20000$. Our detailed abundance analysis for both heavy and light elements are based on higher resolution spectra. The star is found to be very metalpoor with a metallicity $(\sim-2.0)$. Carbon is enhanced with $[\mathrm{C} / \mathrm{Fe}] \sim 1.50$. The heavy s-process element barium is also enhanced. However, as we could not estimate the Eu abundance, its membership for CEMP-r/s class of objects could not be examined based on the CEMP stars classification scheme of Beers \& Christlieb (2005). A comparison of our elemental abundance ratios with literature values including Vanture's are presented in Table 8. Although our $\mathrm{T}_{\text {eff }}$ estimate is close to Vanture's, $\log g$ value differs from his. While for La and Ce our estimates are lower than Vanture's, for $\mathrm{Nd}$ and $\mathrm{Sm}$ our estimates are higher. Spectrum synthesis calculation of La II $4921.77 \AA$ gives a better fit with our adopted La abundance than that obtained using Vanture's atmospheric parameters and abundance value of $\mathrm{La}$. In the $\log \left({ }^{12} C /{ }^{13} C\right)$ versus [C/N] plot (Fig. 20), CD-38 2151 falls in the region occupied by the CEMP stars. Estimated $\mathrm{C} / \mathrm{O}$ $\sim 1.26$ is similar to values seen in $\mathrm{CH}$ stars.

HD 30443 - This star belongs to the CH star catalogue of Bartkevicius (1996). Yamashita (1975) also had classified this object as a $\mathrm{CH}$ star. This work presents for the first time a detailed abundance analysis for this object. The estimated metallicity is -1.68 . The star is enhanced in Carbon with $[\mathrm{C} / \mathrm{Fe}] \sim 1.68$ as well as in other heavy elements. The abundance estimates of the star are characteristic of $\mathrm{CH}$ giants. The estimated $\mathrm{C} / \mathrm{O} \sim 1.02$ is similar to values seen in $\mathrm{CH}$ stars. From the kinematic analysis, HD 30443 is found to be a thin disk star with a probability of 98 percent. The estimated spatial velocity is $\sim 62.04 \mathrm{~km} \mathrm{~s}^{-1}$, which is similar to that expected for thin disk objects (Chen et al. 2004).

HD 29370 and HD 123701 - These two objects are listed in both the CH star catalogue of Bartkevicius (1996) and the barium star catalogue of Lü (1991). Sleivyte \& Bartkevicius (1990) based on photometric analysis included HD 29370 in the category of metal-deficient barium stars. However, Catchpole, Robertson \& Warren (1977) classified HD 29370 as a metal weak CH star. Mennessier et al. (1997) suggested this object to be either a subgiant or a giant star.

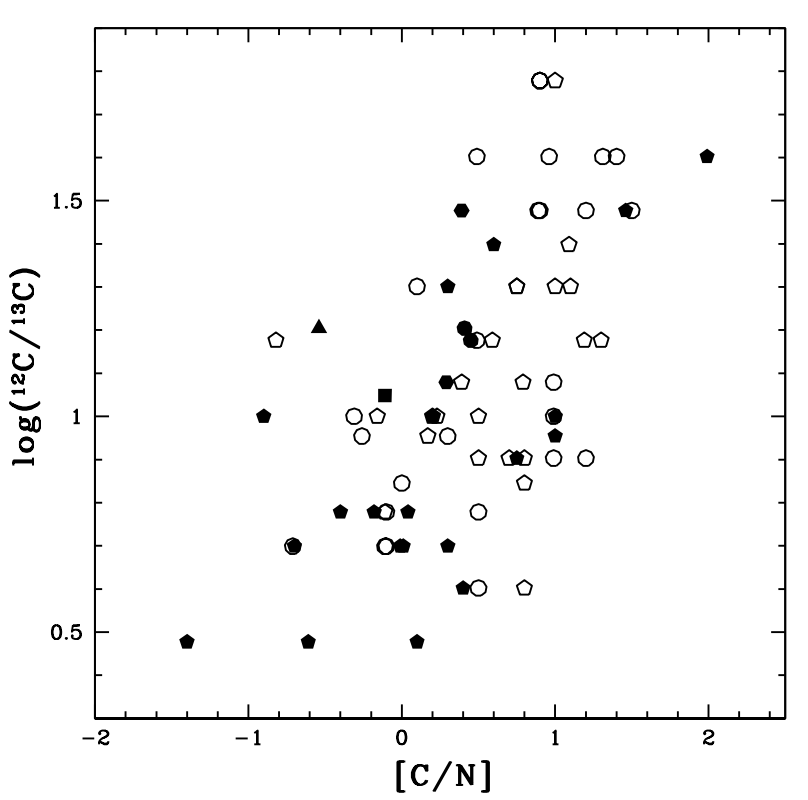

Figure 20. $\log \left({ }^{12} C /{ }^{13} C\right)$ versus $[\mathrm{C} / \mathrm{N}]$ for CEMP stars is presented. The data are taken from Masseron et al. (2010). Open circles represent CEMP-s stars, open pentagon corresponds to CEMP-r/s stars and CEMP-r stars are represented by filled circle. Filled pentagon corresponds to CEMP-no stars and CEMP-low s stars are represented by filled hexagon. CD-28 1082 is represented by a filledd triangle and CD-38 2151 by a filled square.

From our estimates of luminosity and temperature, we have confirmed it to be a giant star from its location in the H-R diagram. de Castro et al. (2016) have estimated the abundance of the $\mathrm{Na}, \mathrm{Al}, \alpha$-elements, iron-peak elements, and s-process elements Y, Zr, La, Ce, and Nd for these two objects. In addition to these elements we have derived abundances for elements C, N and O for HD 123701 and C and $\mathrm{N}$ for HD 29370 which were not presented in de Castro et al. (2016). Our analysis confirms the object HD 123701 to be a barium star with $\mathrm{C} / \mathrm{O} \sim 0.15$. As the oxygen abundance could not be estimated for HD 29370, it could not be classified based on estimates of the $\mathrm{C} / \mathrm{O}$ ratio.

HD 50264 - This object is listed in both the CH star catalogue of Bartkevicius (1996) and the barium star catalogue of Lü (1991). While Bond (1974) considered this star as a $\mathrm{CH}$ subgiant, Mennessier et al. (1997) grouped HD 50264 as a dwarf. According to the analysis of Pereira \& Junqueira (2003) both HD 50264 and HD 87080 are $\mathrm{CH}$ subgiants. Our analysis shows the object to exhibit characteristic properties of barium stars with $\mathrm{C} / \mathrm{O}$ ratio $\sim 0.41$.

HD 87080 - This object is listed in both the $\mathrm{CH}$ star catalogue of Bartkevicius (1996) and the barium star catalogue of Lü (1991). Mennessier et al. (1997) assigned HD 87080 to group of subgiants. Our work confirms the same. A comparison of our estimates of elemental abundance ratios with those of Allen \& Barbuy (2006a,b) is presented in Table 8. For most of the elements we find a good agreement. This object shows the characteristic properties of barium stars with $\mathrm{C} / \mathrm{O}$ ratio $\sim 0.29$.

HD 87853 - This star is found to be mildly metalpoor with an estimated metallicity of -0.73 . All the elements 
show abundances as expected for normal metal-poor star of similar metallicity. This is true for $\alpha$-elements as well as for heavy neutron capture elements. The position of the star on the HR diagram indicates that the star has just left the main-sequence, approaching the subgiant stage.

HD 176021 - Bond (1970) considered this star as a subgiant $\mathrm{CH}$ star. This star belongs to the $\mathrm{CH}$ star catalogue of Bartkevicius (1996). While Bensby, Feltzing \& Oey (2014) derived abundances for a few elements, Battistini \& Bensby (2015) determined the abundances of Sc, V, Mn and Co to study the origin and evolution of these elements in a homogenous sample of stars. According to our abundance estimates, the star qualifies to be a sub-giant $\mathrm{CH}$ star. We have also measured abundances of carbon and nitrogen, and both are found to be similarly enhanced. The ratio of $\mathrm{C} / \mathrm{O}$ could not be estimated for this object.

HD 188985 - This object is listed in both the $\mathrm{CH}$ star catalogue of Bartkevicius (1996) and the barium star catalogue of Lü (1991). North et al. (1994) and Mennessier et al. (1997) placed this object as a barium dwarf. Our analysis confirms the same. Our abundance estimates match closely with those of Allen \& Barbuy (2006a,b). Estimated C/O ratio for this object is $\sim 0.89$.

HD 202020 - From radial velocity monitoring, McClure (1997) confirmed HD 202020 to be a binary with an orbital period of $2064 \pm 10 \mathrm{~d}$. The first abundance analysis for this star was performed by Luck \& Bond (1991); however abundances of elements such as carbon, Nitrogen, Strontium and Barium were not presented. We have estimated the abundances of these elements in the present work. HD 202020 is listed in Bartkevicius (1996). There is a discrepancy regarding the oxygen abundance in this star. If we consider the value of oxygen abundance obtained using the line at $6300.3 \AA$, estimated $\mathrm{C} / \mathrm{O}$ ratio turns out to be $>1$, a value generally seen in case of $\mathrm{CH}$ stars. With $\mathrm{C} / \mathrm{O} \sim 1.99$, the star qualifies to be a $\mathrm{CH}$ star. We get a value much lower than one, if we consider the non-LTE corrected abundance value of oxygen obtained from the oxygen triplet lines. We note that, a value of $\mathrm{C} / \mathrm{O}<1$ is generally seen for barium stars.

The position of our programme stars in the colourmagnitude diagram is shown in Fig. 21. A comparison of the abundances of both light and heavy elements of our programme stars with those of Ba stars, $\mathrm{CH}$ stars and CEMP stars from literature is shown in Figs 22 and 23, respectively.

\section{CONCLUSION}

Abundance analysis results of 12 potential $\mathrm{CH}$ star candidates, 11 from the $\mathrm{CH}$ stars catalogue of Bartkevicious (1996) and one object HE 0308-1612 from Goswami et al. (2010) are presented. Detailed analysis shows two of them, CD-28 1082 and CD-38 2151 to be very metal-poor and highly enhanced in carbon. Five objects HE 0308-1612, HD 30443, HD 87853, HD 176021 and HD 202020 show characteristic properties of $\mathrm{CH}$ stars. Estimated $\mathrm{C} / \mathrm{O}$ ratios for these objects are similar to those generally noticed in $\mathrm{CH}$ stars. While HE 0308-1612 and HD 30443 are CH giants, HD 87853 and HD 176021 are CH subgiants, and HD 202020 is in turn off stage. The abundance patterns of these stars

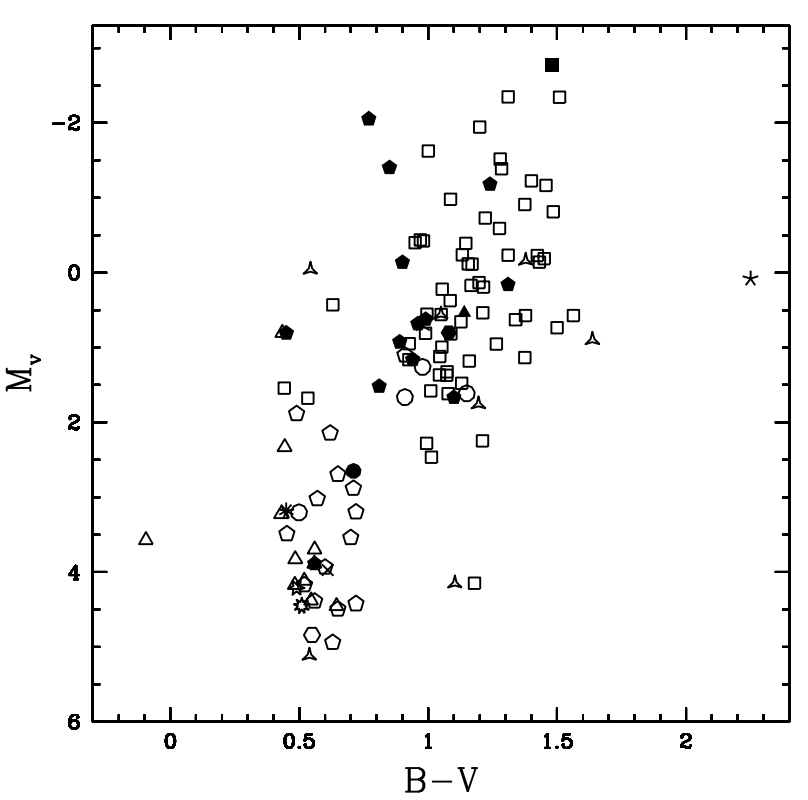

Figure 21. The locations of our programme stars, CD-28 1082 (filled triangle), CD-38 2151 (filled square), HD 50264 (Open hexagon), HD 87080 (filled circle), HD 123701 (skelton triangle), HD 176021 (cross), HD 188985 (star), HD 29370 (filled hexagon), HD 30443 (five sided cross), HE 0308-1612 (six sided cross), HD 87853 (nine sided cross), and HD 202020 (nine sided star), in the colour-magnitude diagram. Open squares represent Ba giants, open triangle is for $\mathrm{Ba}$ dwarfs and open circle represents barium subgiants from literature (de Castro et al. 2016; Yang et al. 2016; Allen \& Barbuy 2006). CH subgiants are represented by open pentagon and filled pentagon represents $\mathrm{CH}$ giants from literatures (Karinkuzhi \& Goswami 2014,2015; Goswami et al. 2016). CEMP stars are represented by starred triangle (Masseron et al. 2010).

match well with the abundance patterns of $\mathrm{CH}$ stars from literature.

While HE 0308-1612, and HD 176021 show moderate enhancement of carbon, HD 202020 shows near solar carbon abundance. Among the $\mathrm{CH}$ stars, HD 30443 shows the highest carbon enhancement with $[\mathrm{C} / \mathrm{Fe}] \sim 1.68$. The remaining five objects, HD 29370, HD 50264, HD 87080, HD 123701 and HD 188985 are found to show the characteristic properties of barium stars. Among the barium stars, only one object HD 87080 is found to be moderately metal-poor $([\mathrm{Fe} / \mathrm{H}]=-0.47)$, the rest four are near solar. Barium stars with $[\mathrm{Ba} / \mathrm{Fe}]>0.60$ are classified as strong Ba stars (Yang et al. 2016). All the Ba stars in our sample show $[\mathrm{Ba} / \mathrm{Fe}]$ $>1$ and are $\mathrm{Ba}$ strong. The abundance patterns of the $\mathrm{Ba}$ stars match well with the abundance patterns of strong $\mathrm{Ba}$ stars in the sample of de Castro et al. (2016) and Yang et al. (2016). de Castro et al. (2016) shows that 90 percent of the $\mathrm{Ba}$ stars in their sample belong to thin disk population. From kinematic analysis, all the five Ba stars are found to belong to thin disk population.

We could estimate the carbon isotopic ratio ${ }^{12} \mathrm{C} /{ }^{13} \mathrm{C}$ only for six objects, two CEMP, two $\mathrm{CH}$ and two barium stars. The values are found to be in the range 7.4-16. The stars for which we could estimate ${ }^{12} \mathrm{C} /{ }^{13} \mathrm{C}$ are either in the giant or the subgiant phase of evolution. During the $\mathrm{H}$ burn- 
Table 8 : Comparison of the abundances of our programme stars with the literature values.

\begin{tabular}{|c|c|c|c|c|c|c|c|c|}
\hline Star name & {$[\mathrm{Fe} \mathrm{I} / \mathrm{H}]$} & {$[\mathrm{Fe} \mathrm{II} / \mathrm{H}]$} & {$[\mathrm{Fe} / \mathrm{H}]$} & {$[\mathrm{Sr} / \mathrm{Fe}]$} & {$[\mathrm{Y} / \mathrm{Fe}]$} & {$[\mathrm{Zr} / \mathrm{Fe}]$} & {$[\mathrm{Ba} \mathrm{II} / \mathrm{Fe}]$} & Ref \\
\hline HE $0308-1612$ & -0.72 & -0.73 & -0.73 & 1.20 & 1.13 & - & 1.63 & 1 \\
\hline CD-28 1082 & -2.46 & -2.44 & -2.45 & 1.44 & 1.61 & - & 2.09 & 1 \\
\hline \multirow[t]{2}{*}{ HD 29370} & -0.15 & -0.13 & -0.14 & 0.82 & 0.87 & 1.64 & 1.53 & 1 \\
\hline & -0.25 & -0.28 & -0.27 & - & 0.83 & 0.71 & - & 2 \\
\hline HD 30443 & -1.68 & -1.69 & -1.68 & 0.74 & 1.37 & 1.60 & 1.70 & 1 \\
\hline \multirow[t]{2}{*}{ CD-38 2151} & -2.03 & -2.03 & -2.03 & 1.16 & 0.57 & 2.00 & 0.97 & 1 \\
\hline & - & - & -1.40 & - & 0.30 & - & - & 3 \\
\hline \multirow[t]{2}{*}{ HD 50264} & -0.14 & -0.11 & -0.13 & 1.90 & 1.16 & - & 1.40 & 1 \\
\hline & -0.34 & -0.37 & -0.34 & - & 0.88 & 1.29 & 1.25 & 4 \\
\hline \multirow[t]{3}{*}{ HD 87080} & -0.47 & -0.49 & -0.48 & 1.84 & 1.12 & - & 1.61 & 1 \\
\hline & -0.51 & -0.49 & -0.51 & - & 1.01 & 1.22 & 1.51 & 4 \\
\hline & -0.49 & -0.44 & - & 0.98 & 1.11 & 1.35 & 1.48 & 5 \\
\hline HD 87853 & -0.72 & -0.74 & -0.73 & - & -0.48 & - & -0.43 & 1 \\
\hline \multirow{2}{*}{ HD 123701} & 0.05 & 0.07 & 0.06 & 1.58 & 1.17 & 0.83 & 1.62 & 1 \\
\hline & -0.44 & -0.46 & -0.45 & - & 0.72 & 0.94 & - & 2 \\
\hline \multirow[t]{2}{*}{ HD 176021} & -0.62 & -0.65 & -0.64 & 1.89 & 1.11 & - & 1.35 & 1 \\
\hline & - & - & -0.40 & 0.95 & 0.75 & 0.44 & 1.22 & 6 \\
\hline \multirow[t]{3}{*}{ HD 188985} & 0.01 & 0.01 & 0.01 & 2.07 & 1.13 & - & 1.46 & 1 \\
\hline & -0.25 & -0.30 & - & 1.05 & 1.02 & 1.18 & 1.20 & 5 \\
\hline & -0.51 & -0.32 & - & 1.10 & 0.95 & 0.85 & 1.27 & 7 \\
\hline \multirow[t]{2}{*}{ HD 202020} & 0.04 & 0.08 & 0.06 & 1.82 & 1.00 & 0.48 & 0.80 & 1 \\
\hline & -0.14 & -0.07 & -0.13 & - & 0.80 & 0.25 & - & 8 \\
\hline Star name & {$[\mathrm{La} \mathrm{II} / \mathrm{Fe}]$} & {$[\mathrm{Ce} \mathrm{II} / \mathrm{Fe}]$} & {$[\mathrm{Pr} \mathrm{II} / \mathrm{Fe}]$} & {$[\mathrm{NdII} / \mathrm{Fe}]$} & {$[\mathrm{Sm} \mathrm{II/Fe]}$} & {$[\mathrm{Eu} \mathrm{II} / \mathrm{Fe}]$} & & Ref \\
\hline HE $0308-1612$ & 1.43 & 1.71 & 2.01 & 1.73 & 1.45 & 0.35 & & 1 \\
\hline CD-28 1082 & 1.55 & 1.97 & 2.30 & 1.99 & 2.29 & 2.07 & & 1 \\
\hline \multirow[t]{2}{*}{ HD 29370} & 1.13 & 1.14 & - & 1.19 & 1.41 & - & & 1 \\
\hline & 1.61 & 1.03 & - & 1.00 & - & - & & 2 \\
\hline HD 30443 & - & 1.62 & 2.67 & 2.47 & 2.24 & - & & 1 \\
\hline \multirow[t]{2}{*}{$\mathrm{CD}-382151$} & 0.88 & 1.24 & 1.52 & 1.34 & 1.53 & - & & 1 \\
\hline & 1.30 & 1.35 & - & 1.00 & 0.61 & - & & 3 \\
\hline \multirow[t]{2}{*}{ HD 50264} & 1.30 & 1.66 & - & 1.35 & - & - & & 1 \\
\hline & 0.92 & 0.76 & - & 0.47 & - & 0.44 & & 4 \\
\hline \multirow[t]{3}{*}{ HD 87080} & 1.75 & 1.71 & 1.68 & 1.47 & 1.68 & 0.36 & & 1 \\
\hline & 1.75 & 1.32 & - & 0.97 & - & 0.61 & & 4 \\
\hline & 1.74 & 1.73 & 1.24 & 1.56 & 1.12 & 0.66 & & 5 \\
\hline HD 87853 & - & - & - & 0.94 & - & 0.34 & & 1 \\
\hline \multirow[t]{2}{*}{ HD 123701} & 1.46 & 1.73 & 1.79 & 1.62 & 1.68 & 0.15 & & 1 \\
\hline & 1.56 & 1.20 & - & 1.14 & - & - & & 2 \\
\hline \multirow[t]{2}{*}{ HD 176021} & 1.33 & 1.47 & - & 1.32 & 1.50 & 0.38 & & 1 \\
\hline & 0.48 & 0.52 & 0.87 & 0.62 & 0.53 & 0.14 & & 6 \\
\hline \multirow[t]{3}{*}{ HD 188985} & 1.39 & 1.56 & - & 1.37 & - & 0.10 & & 1 \\
\hline & 1.16 & 1.23 & 0.82 & 1.04 & 0.72 & 0.29 & & 5 \\
\hline & - & 0.93 & - & 0.89 & - & - & & 7 \\
\hline \multirow[t]{2}{*}{ HD 202020} & 0.75 & 0.82 & - & 0.71 & - & 0.07 & & 1 \\
\hline & 0.67 & 0.03 & 0.29 & 0.25 & -0.04 & - & & 8 \\
\hline
\end{tabular}

Note. References: 1. Our work, 2. de Castro (2016), 3. Vanture(1992), 4. Pereira \& Junqueira (2003), 5. Allen \& Barbuy (2006a), 6. Sneden \& Bond (1976), 7. North et al. (1994b), 8 Luck \& Bond (1991)

ing stage of the main-sequence stars, proton capture of a ${ }^{12} \mathrm{C}$ can take place leading to the production of ${ }^{13} \mathrm{C}$. According to the standard evolutionary theory, the convective envelope of the star, which is ascending the giant branch, can expand inward and mix the CNO processed material from the burning shell to the outer envelope. As a result of this first dredge up, the carbon isotopic ratio on the surface of the star may decrease. This may explain the low ${ }^{12} \mathrm{C} /{ }^{13} \mathrm{C}$ values estimated for our programme stars. The low values of ${ }^{12} \mathrm{C} /{ }^{13} \mathrm{C}(<15)$ may also be explained by certain nucleosynthesis of the material contributed by the donor star, in which the accreted material is mixed into the hydrogen burning region either during the main-sequence or the first ascent of the giant branch (Vanture, 1992).

Nitrogen is enhanced in all the stars other than HD 29370. Nitrogen production is believed to be mostly through CN cycling. First dredge up brings the CNO processed nitrogen to the surface as the star evolves to the red giant branch. Because of this, in normal giants the nitrogen is found to be enhanced and ${ }^{12} \mathrm{C} /{ }^{13} \mathrm{C}$ reduced. A second distinct mixing episode can happen when the star becomes brighter than the RGB bump (Gratton et al. 2000). This can also reduce the observed ${ }^{12} \mathrm{C} /{ }^{13} \mathrm{C}$ ratio to nearly $6-10$ and the abundance of nitrogen can raise by nearly a fac- 

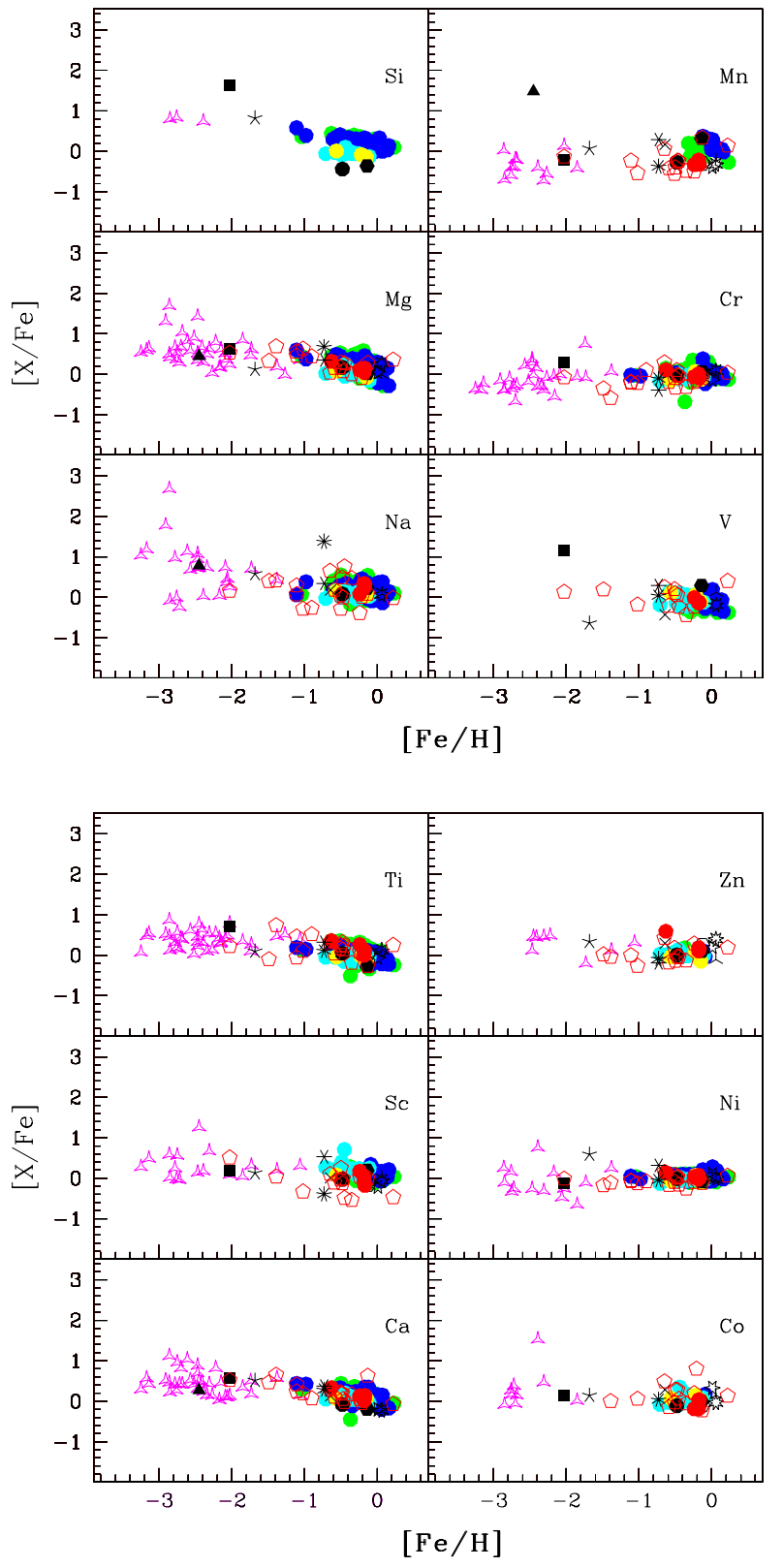

Figure 22. Abundance ratios of light elements with respect to metallicity $[\mathrm{Fe} / \mathrm{H}]$ observed in our programme stars are represented in different symbols. CD-28 1082 (filled triangle), CD-38 2151 (filled square), HD 50264 (Open hexagon), HD 87080 (filled circle), HD 123701 (skelton triangle), HD 176021 (cross), HD 188985 (star), HD 29370 (filled hexagon), HD 202020 (nine sided star), HD 30443 (five sided cross), HE 0308-1612 (six sided cross) and HD 87853 (nine sided cross). Green filled circles represent strong $\mathrm{Ba}$ giants, blue filled circles represent weak Ba giants, cyan filled circles are for $\mathrm{Ba}$ dwarfs and yellow filled circles represent barium subgiants from literature (de Castro et al. 2016; Yang et al. 2016; Allen \& Barbuy 2006). CH subgiants are represented by red filled circles, and red open pentagons represent $\mathrm{CH}$ giants from literature (Karinkuzhi \& Goswami 2014,2015; Goswami et al. 2016). CEMP stars are represented by magenta starred triangle (Masseron et al. 2010).
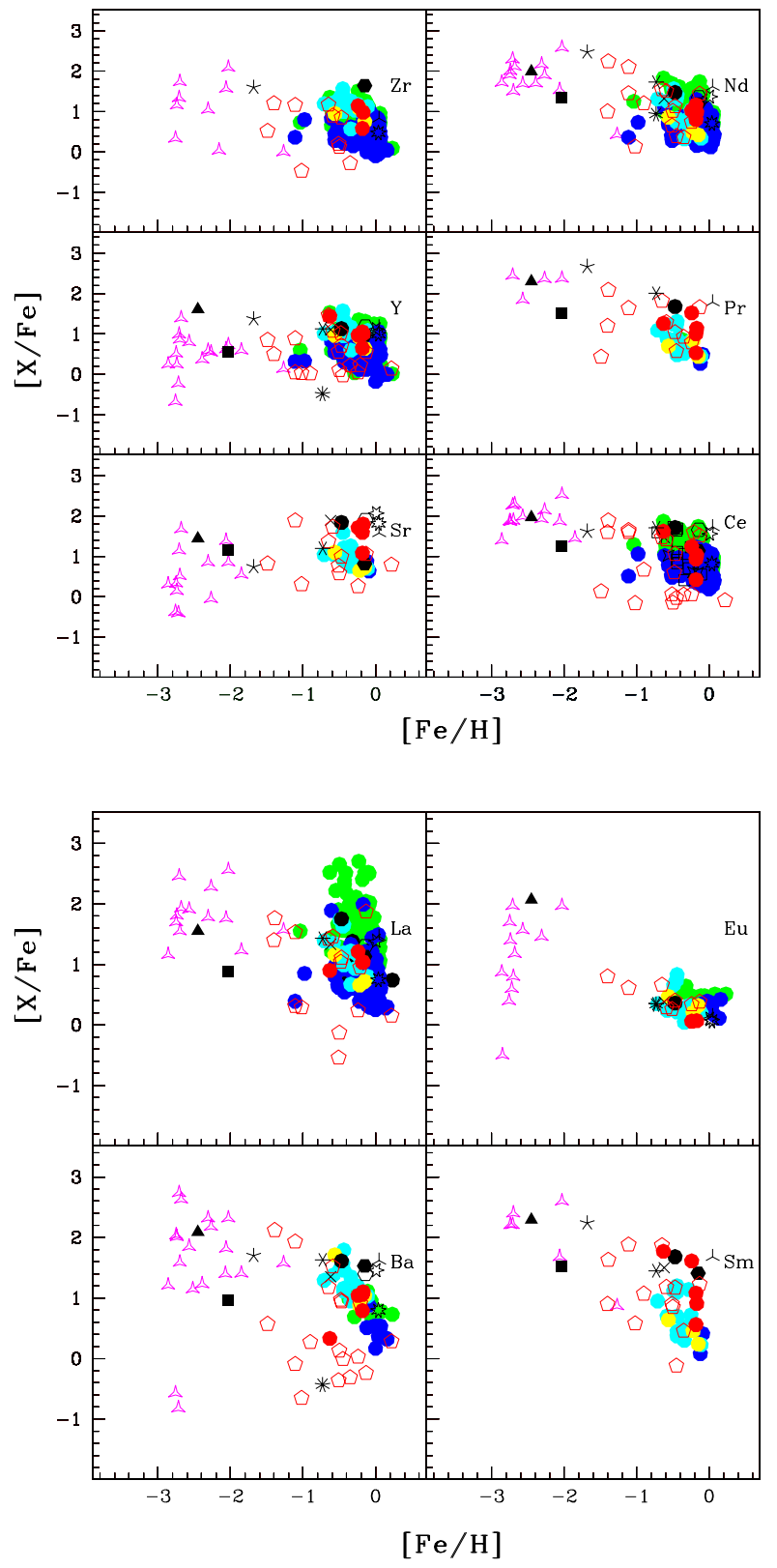

Figure 23. Same as Figure 22 for heavy elements.

tor of 4 . The abundance pattern is expected to be the same until the AGB phase of evolution where the second/third dredge-up can cause changes in the surface chemical compositions. Contrary to this, observations show that the abundances of light elements such as $\mathrm{C}, \mathrm{N}, \mathrm{O}$ and the carbon isotopic ratio are modified at the RGB bump. This is exhibited by more than 95 percent of the low-mass stars and it is found to be independent of the stellar environment (Charbonnel \& do Nascimento, 1998). Zahn (1992) proposed that the interaction between meridional circulation and turbulence induced rotation can be the cause of mixing and the subsequent changes in the surface chemical compositions. Charbonnel (2005) suggested that this extra mixing may be rotation induced. But none of these processes alone could explain the observations. Based on the $3 \mathrm{D}$ modelling of low- 
mass RGB stars (Dearborn, Lattanzio \& Eggleton 2006), Eggleton, Dearborn \& Lattanzio (2006) suggested that the molecular-weight inversion created by ${ }^{3} \mathrm{He}\left({ }^{3} \mathrm{He}, 2 \mathrm{p}\right){ }^{4} \mathrm{He}$ reaction in the outer regions of $\mathrm{H}$ burning shell can cause a transport mechanism often referred as thermohaline mixing that may cause a change in the surface chemical compositions of $\mathrm{C}, \mathrm{N}, \mathrm{O}$, and $\mathrm{Na}$. The effect of thermohaline mixing on the surface abundances of a low-metallicity, low-mass star that has accreted material from a carbon rich companion has also been investigated by Stancliffe et al. (2007) based on binary models. It is commonly assumed that a star that accreted material keep it on the surface without any mixing with the interior until the first dredge up; however, thermohaline mixing can happen that causes the mixing of accreted material with the rest of the star as it has a higher mean molecular weight.

All the stars in our sample show enhancement in heavy elements. As their evolutionary status does not support the observed enhanced abundances of heavy elements, the enhancement may be attributed to an invisible binary companion. In our sample, only HD 202020 is a confirmed binary (McClure 1997).

The abundance results presented here will add to the abundance data obtained from our previous studies in a homogeneous manner and compiled in Cristallo et al. (2016), that can be used to constrain the physics and the nucleosynthesis occuring in low-mass AGB stars. Abundance results of CEMP-r/s stars will provide observational constrains for theoretical studies of i-process, suggested in the recent years for the origin of CEMP-r/s stars.

\section{ACKNOWLEDGEMENT}

We thank the staff at IAO and at the remote control station at CREST, Hosakote, and the staff at VBO, Kavalur, for assisting during the observations. Funding from DST SERB project No. EMR/2016/005283 is gratefully acknowledged. We thank the anonymous referee for many constructive suggestions. We would like to acknowledge Abha $\mathrm{K}$ for performing some initial calculations on a few stars while at IIA during 2015. This work made use of the SIMBAD astronomical database, operated at CDS, Strasbourg, France, the NASA ADS, USA and data from the European Space Agency (ESA) mission Gaia (https://www.cosmos.esa.int/gaia), processed by the Gaia Data Processing and Analysis Consortium (DPAC, https://www.cosmos.esa.int/web/gaia/dpac/consortium).

T.M. acknowledges support provided by the Spanish Ministry of Economy and Competitiveness (MINECO) under grant AYA-2017-88254-P. Based on data collected using VBT echelle, HESP, and FEROS.

\section{REFERENCES}

Abate C., Pols O.R., Izzard R.G., Mohamed S.S., de Mink S. E. 2013, A\&A, 552, A26

Abate C., Pols O.R., Izzard R.G., Karakas A.I., 2015a, A\&A, 581, A22

Abate C., Pols O.R., Karakas A.I., Izzard R.G., 2015b, A\&A, 576, A118

Abate C., Stancliffe R.J., Liu Z-W., 2016, A\&A, 587, 50
Allen D.M., Barbuy B., 2006a, A\&A, 454, 895

Allen D.M., Barbuy B., 2006b, A\&A, 454, 917

Alonso A., Arribas S., Martinez-Roger C., 1999, A\&AS, 140, 261

Alonso A., Arribas S., Martinez-Roger C., 2001, A\&A, 376, 1039

Anderson E.M., Zilitis V.A., Sorokina E.S., 1967, Opt. Spectr. 23, 102-105.

Aoki W., Beers T.C., Christlieb N., Norris J.E., Ryan S.G., Tsangarides S., 2007, ApJ, 655, 492

Argast D., Samland M., Thielemann F.-K., Qian Y.-Z., 2004, A\&A, 416, 997

Arnesen A., Bengtsson A., Hallin R., Lindskog J., Nordling C., Noreland T., 1977, Phys.Scripta 16, 31-34

Asplund M., Grevesse N., Sauval A.J., 2009, Ann. Rev. Astron. Astrophy., 47:481

Bartkevicious A., 1996, Baltic Astron, 5, 217

Battistini C, Bensby T, 2015, A\&A, 577, 9

Beers T.C., Christlieb N., 2005, ARA\&A, 43, 531

Belczynski K., Kalogera V., Bulik T., 2002, ApJ, 572, 407

Bensby T., Feltzing S., Lundström I., 2003, A\&A, 410, 527

Bensby T., Feltzing S., Lundström I., 2004, A\&A, 415, 155

Bensby T., Feltzing S., Oey M.S., 2014, A\&A, 562, 71

Biemont E., Grevesse N., Hannaford P., Lowe R.M., 1981, ApJ $248,867-873$

Biemont E., Karner C., Meyer G., Traeger F., zu Putlitz G. 1982, A\&A 107, 166-171

Bisterzo S., Gallino R., Straniero O., Cristallo S., Käppeler F., 2011, MNRAS, 418, 284

Bond H.E., 1970, ApJS, 22, 117B

Bond H.E., 1974, ApJ, 194, 95

Catchpole R.M., Robertson B.S.C., Warren P.R., 1977, MNRAS, 181,391

Charbonnel C., 2005, ASP Conference Series, Vol. 336

Charbonnel C., do Nascimento J., 1998, A\&A, 336, 915

Chen B., Vergely J.L., Valette B., Carraro G., 1998, A\&A, 336, 137

Chen Y.Q., Nissen P.E., Zhao G., 2004, A\&A, 425, 697

Christlieb N., Green P. J., Wisotzki L., Reimers D., 2001, A\&A, 375,366

Cohen J.G., Christlieb N., Qian Y.Z., Wasserburg G.J., 2003, AJ, 588,1082

Corliss C.H., Bozman W.R., 1962, NBS Monograph 53

Cowley C.R., Corliss C.H., 1983, MNRAS 203, 651-659

Cristallo S., Karinkuzhi D., Goswami A., Piersanti L., Gobrecht D., 2016, ApJ, 833, 181

Cutri R.M., Skrutskie M.F., Van Dyk S., Beichman C A., Carpenter J.M, 2003, yCat.2246.0

de Castro D.B., Pereira C.B., Roig F., Jilinski E., Drake N.A., Chavero C., Sales Silva J.V., 2016, MNRAS, 459, 4299

Dearborn D. S. P., Lattanzio J. C., Eggleton P. P., 2006, ApJ, 639,405

Edvardsson B., Anderson J., Gustafsson B., Lambert D.L., Nissen P.E., Tomkin, J., 1993, A\&A,275, 101

Eggen O.J., 1997, AJ, 114, 825

Eggleton P. P., Dearborn D. S. P., Lattanzio J. C., 2006, Science, 314, 1580

Eriksson K., Toft S.C., 1979, A\&A, 71, 178

Frischknecht U. et al., 2016, MNRAS, 456, 1803

Führ J.R., Martin G.A., Wiese W.L., 1988, J. Phys. Chem. Ref. Data 17, Suppl. 4

Garz T., 1973, A\&A. 26, 471.

Girardi L., Bressan A., Bertelli G., Chiosi C., 2000, A\&AS 141, 371

Goswami A., 2005, MNRAS, 359, 531

Goswami A., Aoki W., Beers T.C., Christlieb N., Norris J.E., Ryan S.G., Tsangarides S., 2006, MNRAS, 372, 343

Goswami A., Bama P., Shantikumar N.S., Devassy D., 2007, BASI, 35, 339

Goswami A., Aoki W., 2010, MNRAS, 404, 253 
Goswami A., Karinkuzhi D., Shantikumar N.S., 2010, MNRAS, 402, 1111

Goswami A., Aoki W., Karinkuzhi D., 2016, MNRAS, 455, 402

Gratton R.G., Sneden C., 1988, A\&A, 204, 193

Gratton R.G., Sneden C., Carretta E., Bragaglia A., 2000, A\&A, 354,169

Hampel M., Stancliffe R.J., Lugaro M., Meyer B.S., 2016, ApJ, 831,171

Hannaford P., Lowe R.M., Grevesse N., Biemont E., Whaling W., 1982, ApJ 261, 736-746

Hansen T.T., Andersen J., Nordström B., Beers T C., Placco V M., Yoon J., Buchhave L.A., 2016, A\&A, 588, 3

Haynes C.J., Kobayashi C., 2019, MNRAS, 483, 5123

Heise H., 1974, A\&A 34, 275

Johnson D.R.H., Soderblom D.R., 1987, AJ, 93, 864

Jonsell K., Barklem P.S., Gustafsson B., Christlieb N., Hill V., Beers T. C., Holmberg J., 2006, A\&A, 451, 651

Jorissen A., Eck S.V., Winckel H.V., Merle T., Boffin H.M.J., Andersen J., Nordström B., Udry S. et al., 2016, A\&A, 586, 158

Karinkuzhi D., Goswami A., 2014, MNRAS, 440, 1095

Karinkuzhi D., Goswami A., 2015, MNRAS, 446, 2348

Keenan P.C., 1942, ApJ, 96, 101

Kurucz R.L., Peytremann E., 1975, SAO Special Report 362

Kurucz R.L., 1988, Trans. IAU, XXB, M. McNally, ed., Dordrecht: Kluwer, 168-172

Lage C.S., Whaling W., 1976, JQSRT 16, 537-542

Lambert D.L., Heath J.E., Lemke M., Drake J., 1996, ApJS, 103, 183

Laughlin C., Victor G.A., 1974, ApJ 192, 551

Lincke R., Ziegenbein G., 1971, Z. Phyzik, 241, 369.

Lü P.K., 1991, AJ, 101, 2229

Lucatello S., Tsangarides S., Beers T.C., Carretta E., Gratton R.G., Ryan S.G., 2005, ApJ, 625, 825

Luck R.E., Bond H.E., 1991, ApJS, 77, 515

Magain, 1989, A\&A, 209, 211

Martin G.A., Führ J.R., Wiese W.L., 1988, J.Phys.Chem.Ref.Data, 17, Suppl.3

Masseron T., Johnson J.A., Plez B., Van Eck S., Goriely S., Jorrison A., 2010, A\&A, 509, 93

McClure R.D., 1983, ApJ, 208, 264

McClure R.D., 1984, ApJ, 280, 31

McClure R.D., Woodsworth W., 1990, ApJ, 352, 709

McClure R.D., 1997, PASP, 109, 536

McWilliiam A., 1998, AJ, 115, 1640

Meggers W.F., Corliss C.H., Scribner B.F., 1975, NBS Monograph 145

Mennessier M.O., Luri X., Figueras F., Gomez A.E., Grenier S., Torra J., North P., 1997, A\&A, 326, 722

Mishenina T.V., Soubiran C., Kovtyukh V.V., Korotin S.A., 2004, A\&A, 418, 551

North P., Berthet S., Lanz T., 1994a, A\&A, 281, 775

North P., Berthet S., Lanz T., 1994b, A\&AS, 103, 321

Pereira C.B., Junqueira S., 2003, A\&A, 402, 1061

Prochaska J.X., McWilliam A., 2000, ApJ, 537, 57

Qian Y.-Z., Woosley S.E., 1996, ApJ, 471, 331

Reddy B.E., Lambert D.L., Priesto C.A., 2006, MNRAS, 367, 1329

Rosswog S., Liebendörfer M., Thielemann F.-K., Davies M.B., Benz W., Piran., 1999, A\&A, 341, 499

Rosswog S., Davies M.B., Thielemann F.-K., Piran T., 2000, A\&A, 360, 171

Rosswog S., Davies M.B., 2002, MNRAS, 334, 481

Schönrich R., Binney J., Dehnen W., MNRAS, 2010, 403, 1829

Schulz-Gulde E., 1969, JQSRT 9, 13

Shen S., Cooke R.J., Ramirez-Ruiz E., Madau P., Mayer L., Guedes J., 2015, ApJ, 807, 115

Sleivyte J., Bartkevicius A., 1990, ViloB, 85, 3
Smith P. L., Kuhne M., 1978, Proc. Roy. Soc., 363, 263

Sneden C., 1973, PhD thesis, Univ. Texas

Sneden C., Bond H.E., 1976, ApJ, 204, 810

Stancliffe R.J., Glebbeek E., Izzard R.G., Pols O.R., 2007, A\&A, 464,57

Starkenburg E., Shetrone M D., McConnachie A W., Venn K A., 2014, MNRAS, 441, 1217

Tammann G.A., Löffler W., Schröder, A. 1994, ApJS, 92, 487

Thielemann F.-K. et al., 2002, SSRv, 100, 277

Thielemann F.-K. et al. 2011, PrPNP, 66, 346

Van de Voort F., Quataert E., Hopkins P.F., Keres D., FaucherGiguere C.A., 2015, MNRAS., 447, 140

Vanture A.D., 1992, AJ, 104, 1977

Ward L., Vogel O., Arnesen A., Hallin R., Wannstrom A., 1985, Phys. Scripta 31, 162-165

Warner B., 1968, MNRAS 140, 53-59

Wehmeyer B., Pignatari M., Thielemann F.-K., 2015, MNRAS, 452, 1970

Wiese W.L., Smith M.W., Miles B.M., 1969, NSRDS-NBS 22

Wolnik S.J., Berthel R.O., Carnevale E.H., Wares G.W., 1969, ApJ 157, 983

Woolf V.M., Wallerstein G., MNRAS, 356, 963

Worley C.C., Hill V.J., Sobeck J., Carretta E., 2013, A\&A, 553, A47

Yamashita Y., 1975, PASJ, 27, 325

Yang G.C., Liang Y.C., Spite M., Chen Y.Q., Zhao G. et al., 2016, RAA, 16,1

Zahn J.P., 1992, A\&A, 265, 115

Zijlstra A.A., 2004, MNRAS, 348, 23 
Table A1 : Elemental abundances in HE 0308-1612, CD-28 1082 and HD 29370

\begin{tabular}{|c|c|c|c|c|c|c|c|c|c|c|c|}
\hline & \multirow[b]{2}{*}{$\mathrm{Z}$} & \multirow[b]{2}{*}{ Solar $\log \epsilon^{a}$} & \multicolumn{3}{|c|}{ HE $0308-1612$} & \multicolumn{3}{|c|}{ CD-28 1082} & \multicolumn{3}{|c|}{ HD 29370} \\
\hline & & & $\log \epsilon$ & {$[\mathrm{X} / \mathrm{H}]$} & {$[\mathrm{X} / \mathrm{Fe}]$} & $\log \epsilon$ & {$[\mathrm{X} / \mathrm{H}]$} & {$[\mathrm{X} / \mathrm{Fe}]$} & $\log \epsilon$ & {$[\mathrm{X} / \mathrm{H}]$} & {$[\mathrm{X} / \mathrm{Fe}]$} \\
\hline $\mathrm{C}$ & 6 & 8.43 & 8.48(syn) & 0.05 & 0.78 & 8.16(syn) & -0.27 & 2.19 & 8.37 (syn) & -0.06 & 0.09 \\
\hline $\mathrm{N}$ & 7 & 7.83 & $7.25(\operatorname{syn})$ & -0.58 & 0.15 & $8.10(\operatorname{syn})$ & 0.27 & 2.73 & $7.70($ syn) & -0.13 & 0.02 \\
\hline $\mathrm{O}$ & 8 & 8.69 & - & - & - & - & - & - & - & - & - \\
\hline $\mathrm{Na} \mathrm{I}$ & 11 & 6.24 & $5.85(\operatorname{syn})$ & -0.39 & 0.34 & $5.59(\operatorname{syn})$ & -2.01 & 0.45 & $6.32(1)$ & 0.08 & 0.23 \\
\hline $\mathrm{Mg} \mathrm{I}$ & 12 & 7.60 & $7.22 \pm 0.01(2)$ & -0.38 & 0.35 & 5.59 (syn) & -2.01 & 0.45 & $7.47(1)$ & -0.13 & 0.02 \\
\hline Si I & 14 & 7.51 & - & - & - & - & - & - & $7.00 \pm 0.04(2)$ & -0.51 & -0.36 \\
\hline $\mathrm{Ca} \mathrm{I}$ & 20 & 6.34 & $5.98 \pm 0.18(5)$ & -0.36 & 0.37 & $4.15 \pm 0.12(4)$ & -2.19 & 0.27 & $6.00 \pm 0.02(3)$ & -0.34 & -0.19 \\
\hline Sc II & 21 & 3.15 & $2.95(1)$ & -0.2 & 0.53 & - & - & - & $3.21 \pm 0.16(3)$ & 0.06 & 0.19 \\
\hline Ti I & 22 & 4.95 & $4.54 \pm 0.15(5)$ & -0.41 & 0.32 & - & - & - & $4.52 \pm 0.05(4)$ & -0.43 & -0.28 \\
\hline Ti II & 22 & 4.95 & $4.52(1)$ & -0.43 & 0.30 & - & - & - & $4.54 \pm 0.14(2)$ & -0.41 & -0.28 \\
\hline V I & 23 & 3.93 & 3.49 (syn) & -0.44 & 0.29 & - & - & - & $4.07 \pm 0.14(3)$ & 0.14 & 0.29 \\
\hline Cr I & 24 & 5.64 & $4.53 \pm 0.20(3)$ & -1.11 & -0.39 & - & - & - & $5.54 \pm 0.09(3)$ & -0.10 & 0.05 \\
\hline Cr II & 24 & 5.64 & - & & & - & - & - & $5.33(1)$ & -0.31 & -0.18 \\
\hline Mn I & 25 & 5.43 & $4.99 \pm 0.13(3)$ & -0.44 & 0.29 & $4.45(\mathrm{syn})$ & -0.98 & 1.48 & $5.60($ syn $)$ & 0.17 & 0.32 \\
\hline Fe I & 26 & 7.50 & $6.78 \pm 0.19(40)$ & -0.72 & - & $5.04 \pm 0.08(13)$ & -2.46 & - & $7.35 \pm 0.11(35)$ & -0.15 & - \\
\hline $\mathrm{Fe}$ II & 26 & 7.50 & $6.77 \pm 0.15(3)$ & -0.73 & - & $5.06 \pm 0.02(2)$ & -2.44 & - & $7.37 \pm 0.04(5)$ & -0.13 & - \\
\hline Co I & 27 & 4.99 & - & - & - & - & - & - & $4.83 \pm 0.09(2)$ & -0.16 & -0.01 \\
\hline $\mathrm{Ni} \mathrm{I}$ & 28 & 6.22 & $5.81 \pm 0.13(5)$ & -0.41 & 0.32 & - & - & - & $6.01 \pm 0.13(4)$ & -0.21 & -0.06 \\
\hline Zn I & 30 & 4.56 & $3.65(\operatorname{syn})$ & -0.91 & -0.19 & - & - & - & $4.51(1)$ & -0.05 & 0.10 \\
\hline Sr I & 38 & 2.87 & $3.24(1)$ & 0.37 & 1.1 & $1.85(\mathrm{syn})$ & -1.02 & 1.44 & $3.54(\operatorname{syn})$ & 0.67 & 0.82 \\
\hline Y II & 39 & 2.21 & $2.61 \pm 0.09(4)$ & 0.4 & 1.13 & $1.38(1)$ & -0.83 & 1.61 & $2.95 \pm 0.14(2)$ & 0.74 & 0.87 \\
\hline Zr II & 40 & 2.58 & - & - & - & - & - & - & $4.09(1)$ & 1.51 & 1.64 \\
\hline $\mathrm{Ba}$ II & 56 & 2.18 & 3.08(syn) & 0.9 & 1.63 & $1.83(\mathrm{syn})$ & -0.35 & 2.09 & $3.58(\operatorname{syn})$ & 1.40 & 1.53 \\
\hline La II & 57 & 1.10 & $1.80($ syn $)$ & 0.7 & 1.43 & 0.21 (syn) & -0.89 & 1.55 & $2.10(\mathrm{syn})$ & 1.00 & 1.13 \\
\hline Ce II & 58 & 1.58 & $2.56 \pm 0.08(3)$ & 0.98 & 1.71 & $1.11 \pm 0.07(2)$ & -0.47 & 1.97 & $2.59 \pm 0.11(2)$ & 1.01 & 1.14 \\
\hline $\operatorname{Pr}$ II & 59 & 0.72 & $2.00($ syn $)$ & 1.28 & 2.01 & $0.58(1)$ & -0.14 & 2.30 & - & - & - \\
\hline Nd II & 60 & 1.42 & $2.42 \pm 0.18(10)$ & 1.0 & 1.73 & $0.97 \pm 0.12(7)$ & -0.45 & 1.99 & $2.48 \pm 0.05(3)$ & 1.06 & 1.19 \\
\hline Sm II & 62 & 0.96 & $1.68 \pm 0.14(2)$ & 0.72 & 1.45 & $0.81(1)$ & -0.15 & 2.29 & $2.24(1)$ & 1.28 & 1.41 \\
\hline Eu II & 63 & 0.52 & $0.14(\operatorname{syn})$ & -0.38 & 0.35 & 0.15 (syn) & -0.37 & 2.07 & - & - & - \\
\hline
\end{tabular}

Table A2 : Elemental abundances in HD 30443, CD-38 2151 and HD 50264

\begin{tabular}{|c|c|c|c|c|c|c|c|c|c|c|c|}
\hline & \multirow[b]{2}{*}{$\mathrm{Z}$} & \multirow[b]{2}{*}{ Solar $\log \epsilon^{a}$} & \multicolumn{3}{|c|}{ HD 30443} & \multicolumn{3}{|c|}{ CD-38 2151} & \multicolumn{3}{|c|}{ HD 50264} \\
\hline & & & $\log \epsilon$ & {$[\mathrm{X} / \mathrm{H}]$} & {$[\mathrm{X} / \mathrm{Fe}]$} & $\log \epsilon$ & {$[\mathrm{X} / \mathrm{H}]$} & {$[\mathrm{X} / \mathrm{Fe}]$} & $\log \epsilon$ & {$[\mathrm{X} / \mathrm{H}]$} & {$[\mathrm{X} / \mathrm{Fe}]$} \\
\hline $\mathrm{C}$ & 6 & 8.43 & 8.43(syn) & 0.00 & 1.68 & $7.9($ syn $)$ & -0.53 & 1.50 & $8.50(\operatorname{syn})$ & 0.07 & 0.21 \\
\hline $\mathrm{N}$ & 7 & 7.83 & $6.55(\mathrm{syn})$ & -1.28 & 0.40 & $7.2(\mathrm{syn})$ & -0.63 & 1.40 & $8.35(\operatorname{syn})$ & 0.52 & 0.66 \\
\hline $\mathrm{O}$ & 8 & 8.69 & $8.42(\operatorname{syn})$ & -0.27 & 1.41 & $7.22(\mathrm{syn})$ & -1.47 & 0.56 & $8.89(\operatorname{syn})$ & 0.20 & 0.34 \\
\hline $\mathrm{Na} \mathrm{I}$ & 11 & 6.24 & $5.14 \pm 0.20(3)$ & -1.1 & 0.58 & - & - & - & - & - & - \\
\hline $\mathrm{Mg} \mathrm{I}$ & 12 & 7.60 & $6.04 \pm 0.13(2)$ & -1.56 & 0.12 & $6.20($ syn $)$ & -1.40 & 0.63 & $7.60 \pm 0.10(3)$ & 0.00 & 0.14 \\
\hline Si I & 14 & 7.51 & $6.65 \pm 0.05(2)$ & -0.86 & 0.82 & 7.10(syn) & -0.41 & 1.62 & - & - & - \\
\hline $\mathrm{Ca} \mathrm{I}$ & 20 & 6.34 & $5.17 \pm 0.17(4)$ & -1.17 & 0.51 & $4.89 \pm 0.08(2)$ & -1.45 & 0.58 & $6.08 \pm 0.12(8)$ & -0.26 & -0.12 \\
\hline Sc II & 21 & 3.15 & $1.60(\mathrm{syn})$ & -1.55 & 0.13 & $1.30 \pm 0.12(2)$ & -1.86 & 0.18 & $2.88($ syn $)$ & -0.27 & -0.16 \\
\hline Ti I & 22 & 4.95 & $3.37 \pm 0.16(9)$ & -1.58 & 0.10 & $3.63 \pm 0.06(3)$ & -1.32 & 0.71 & $4.85 \pm 0.06(6)$ & -0.10 & 0.04 \\
\hline Ti II & 22 & 4.95 & $3.68 \pm 0.25(4)$ & -1.27 & 0.41 & $3.21 \pm 0.17(2)$ & -1.74 & 0.29 & $4.80 \pm 0.09(6)$ & -0.15 & -0.04 \\
\hline V I & 23 & 3.93 & 1.61(syn) & -2.32 & -0.64 & $3.06 \pm 0.07(2)$ & -0.87 & 1.16 & - & - & - \\
\hline Cr I & 24 & 5.64 & - & - & - & $3.91 \pm 0.06(5)$ & -1.73 & 0.30 & $5.54 \pm 0.10(9)$ & -0.10 & 0.04 \\
\hline Cr II & 24 & 5.64 & - & - & - & - & - & - & $5.56 \pm 0.06(3)$ & -0.08 & 0.03 \\
\hline Mn I & 25 & 5.43 & $3.88(\operatorname{syn})$ & -1.61 & 0.07 & $3.20($ syn $)$ & -2.23 & -0.20 & $4.99(\mathrm{syn})$ & -0.44 & -0.30 \\
\hline Fe I & 26 & 7.50 & $5.82 \pm 0.05(11)$ & -1.68 & - & $5.47 \pm 0.10(12)$ & -2.03 & - & $7.36 \pm 0.09(93)$ & -0.14 & - \\
\hline $\mathrm{Fe} I I$ & 26 & 7.50 & $5.81 \pm 0.11(3)$ & -1.69 & - & $5.47(1)$ & -2.03 & - & $7.39 \pm 0.07(11)$ & -0.11 & - \\
\hline Co I & 27 & 4.99 & $3.45(\operatorname{syn})$ & -1.54 & 0.14 & $3.11(1)$ & -1.88 & 0.15 & $4.76 \pm 0.02(2)$ & -0.23 & -0.09 \\
\hline Ni I & 28 & 6.22 & $5.13 \pm 0.22(3)$ & -1.09 & 0.59 & $4.06(1)$ & -2.16 & -0.13 & $6.01 \pm 0.17(7)$ & -0.21 & -0.07 \\
\hline Zn I & 30 & 4.56 & $3.22(1)$ & -1.34 & 0.34 & - & - & - & $4.67 \pm 0.06(2)$ & 0.11 & 0.25 \\
\hline Sr I & 38 & 2.87 & 1.93(syn) & -0.94 & 0.74 & $2.00($ syn $)$ & -0.87 & 1.16 & 4.63(syn) & 1.76 & 1.90 \\
\hline Y II & 39 & 2.21 & $1.90 \pm 0.04(2)$ & -0.31 & 1.37 & $0.75 \pm 0.14(2)$ & -1.46 & 0.57 & $3.26 \pm 0.08(7)$ & 1.05 & 1.16 \\
\hline Zr II & 40 & 2.58 & $2.50($ syn $)$ & -0.08 & 1.60 & $2.55(1)$ & -0.03 & 2.00 & - & - & - \\
\hline $\mathrm{Ba}$ II & 56 & 2.18 & $2.20(\mathrm{syn})$ & 0.02 & 1.70 & $1.12(\mathrm{syn})$ & -1.06 & 0.97 & 3.47 (syn) & 1.29 & 1.40 \\
\hline La II & 57 & 1.10 & - & - & - & $-0.05(\mathrm{syn})$ & -1.15 & 0.88 & $2.29(\operatorname{syn})$ & 1.19 & 1.30 \\
\hline Ce II & 58 & 1.58 & $1.52 \pm 0.12(5)$ & -0.06 & 1.62 & $0.79 \pm 0.07(2)$ & -0.79 & 1.24 & $3.13 \pm 0.05(2)$ & 1.55 & 1.66 \\
\hline Pr II & 59 & 0.72 & $1.71 \pm 0.07(4)$ & 0.98 & 2.67 & $0.21(1)$ & -0.51 & 1.52 & - & - & - \\
\hline Nd II & 60 & 1.42 & $2.21 \pm 0.18(9)$ & 0.79 & 2.47 & $0.73 \pm 0.17(3)$ & -0.69 & 1.34 & $2.66 \pm 0.09(4)$ & 1.24 & 1.35 \\
\hline Sm II & 62 & 0.96 & $1.52 \pm 0.17(5)$ & 0.56 & 2.24 & $0.46(1)$ & -0.50 & 1.53 & - & - & - \\
\hline Eu II & 63 & 0.52 & - & - & - & - & - & - & - & - & - \\
\hline
\end{tabular}

a Asplund (2009), The number inside the parenthesis shows the number of lines used for the abundance determination. 
Table A3 : Elemental abundances in HD 87080, HD 87853 and HD 123701.

\begin{tabular}{|c|c|c|c|c|c|c|c|c|c|c|c|}
\hline & \multirow[b]{2}{*}{$\mathrm{Z}$} & \multirow[b]{2}{*}{ Solar $\log \epsilon^{a}$} & \multicolumn{3}{|c|}{ HD 87080} & \multicolumn{3}{|c|}{ HD 87853} & \multicolumn{3}{|c|}{ HD 123701} \\
\hline & & & $\log \epsilon$ & {$[\mathrm{X} / \mathrm{H}]$} & {$[\mathrm{X} / \mathrm{Fe}]$} & $\log \epsilon$ & {$[\mathrm{X} / \mathrm{H}]$} & {$[\mathrm{X} / \mathrm{Fe}]$} & $\log \epsilon$ & {$[\mathrm{X} / \mathrm{H}]$} & {$[\mathrm{X} / \mathrm{Fe}]$} \\
\hline $\mathrm{C}$ & 6 & 8.43 & $8.05(\operatorname{syn})$ & -0.38 & 0.09 & - & - & - & 8.53(syn) & 0.10 & 0.05 \\
\hline $\mathrm{N}$ & 7 & 7.83 & $8.25(\mathrm{syn})$ & 0.42 & 0.89 & - & - & - & 8.75 (syn) & 0.92 & 0.87 \\
\hline $\mathrm{O}$ & 8 & 8.69 & 8.59 (syn) & -0.10 & 0.37 & 8.35(syn) & -0.34 & 0.39 & 9.36(syn) & 0.67 & 0.62 \\
\hline $\mathrm{Na} \mathrm{I}$ & 11 & 6.24 & $5.85 \pm 0.10(2)$ & -0.39 & 0.08 & $5.92(\mathrm{syn})$ & -0.32 & 0.41 & $6.37 \pm 0.01(2)$ & 0.13 & 0.08 \\
\hline $\mathrm{Mg} \mathrm{I}$ & 12 & 7.60 & $7.30 \pm 0.06(2)$ & -0.30 & 0.17 & $7.55(1)$ & -0.05 & 0.68 & $7.64(1)$ & 0.04 & -0.01 \\
\hline Si I & 14 & 7.51 & $6.60 \pm 0.11(3)$ & -0.91 & -0.44 & - & - & - & - & - & - \\
\hline $\mathrm{Ca} \mathrm{I}$ & 20 & 6.34 & $5.79 \pm 0.11(8)$ & -0.55 & -0.08 & $5.90 \pm 0.20(12)$ & -0.44 & 0.29 & $6.14 \pm 0.05(4)$ & -0.20 & -0.25 \\
\hline Sc II & 21 & 3.15 & $2.63(\operatorname{syn})$ & -0.52 & -0.03 & 2.03(syn) & -1.15 & -0.39 & $3.25(\mathrm{syn})$ & 0.10 & 0.03 \\
\hline $\mathrm{Ti} \mathrm{I}$ & 22 & 4.95 & $4.53 \pm 0.13(5)$ & -0.42 & 0.05 & $4.34(1)$ & -0.61 & 0.12 & $5.14 \pm 0.18(7)$ & 0.19 & 0.14 \\
\hline Ti II & 22 & 4.95 & $4.48 \pm 0.09(4)$ & -0.47 & 0.02 & $4.33 \pm 0.12(5)$ & -0.62 & 0.11 & $5.34 \pm 0.20(3)$ & 0.39 & 0.32 \\
\hline V I & 23 & 3.93 & - & - & - & $3.24(1)$ & -0.69 & 0.04 & $4.10 \pm 0.06(3)$ & 0.17 & 0.12 \\
\hline Cr I & 24 & 5.64 & $5.14 \pm 0.08(3)$ & -0.50 & -0.03 & $4.81 \pm 0.05(5)$ & -0.83 & -0.10 & $5.76 \pm 0.09(3)$ & 0.12 & 0.07 \\
\hline Cr II & 24 & 5.64 & $5.07 \pm 0.04(3)$ & -0.57 & -0.08 & $4.77(1)$ & -0.87 & -0.14 & - & - & - \\
\hline Mn I & 25 & 5.43 & 4.69(syn) & -0.74 & -0.27 & 4.33(syn) & -1.10 & -0.37 & $5.25(\operatorname{syn})$ & -0.18 & -0.23 \\
\hline Fe I & 26 & 7.50 & $7.03 \pm 0.09(65)$ & -0.47 & - & $6.78 \pm 0.09(53)$ & -0.72 & - & $7.55 \pm 0.09(38)$ & 0.05 & - \\
\hline Fe II & 26 & 7.50 & $7.00 \pm 0.04(10)$ & -0.49 & - & $6.76 \pm 0.09(2)$ & -0.74 & - & $7.57 \pm 0.09(13)$ & 0.07 & - \\
\hline Co I & 27 & 4.99 & $4.41 \pm 0.09(2$ & -0.58 & -0.11 & $4.30(1)$ & -0.69 & 0.04 & $5.19(1)$ & 0.20 & 0.15 \\
\hline $\mathrm{Ni} \mathrm{I}$ & 28 & 6.22 & $5.74 \pm 0.18(6)$ & -0.48 & -0.01 & $5.45 \pm 0.13(8)$ & -0.77 & -0.04 & $6.22 \pm 0.19(6)$ & 0.0 & -0.05 \\
\hline Zn I & 30 & 4.56 & $4.10(1)$ & -0.46 & 0.01 & $3.75 \pm 0.02(2)$ & -0.81 & -0.08 & $4.50(1)$ & -0.06 & -0.11 \\
\hline Sr I & 38 & 2.87 & $4.24(\operatorname{syn})$ & 1.37 & 1.84 & - & - & - & 4.50 (syn) & 1.63 & 1.58 \\
\hline Y II & 39 & 2.21 & $2.84 \pm 0.07(7)$ & 0.63 & 1.12 & $1.00 \pm 0.05(3)$ & -1.21 & -0.48 & $3.45 \pm 0.14(3)$ & 1.24 & 1.17 \\
\hline Zr II & 40 & 2.58 & - & - & - & - & - & - & $3.48(1)$ & 0.90 & 0.83 \\
\hline Ba II & 56 & 2.18 & 3.30(syn) & 1.12 & 1.61 & $1.02(\operatorname{syn})$ & -1.16 & -0.43 & $3.87(\operatorname{syn})$ & 1.69 & 1.62 \\
\hline La II & 57 & 1.10 & $2.36(\operatorname{syn})$ & 1.26 & 1.75 & - & - & - & 2.63(syn) & 1.53 & 1.46 \\
\hline Ce II & 58 & 1.58 & $2.80 \pm 0.04(4)$ & 1.22 & 1.71 & - & - & - & $3.38 \pm 0.04(4)$ & 1.80 & 1.73 \\
\hline Pr II & 59 & 0.72 & - & - & - & - & - & - & $2.58(1)$ & 1.86 & 1.79 \\
\hline Nd II & 60 & 1.42 & $2.40 \pm 0.15(10)$ & 0.98 & 1.47 & $1.63(1)$ & 0.21 & 0.94 & $3.11 \pm 0.15(5)$ & 1.69 & 1.62 \\
\hline Sm II & 62 & 0.96 & $2.15 \pm 0.17(3)$ & 1.19 & 1.68 & - & - & - & $2.71(1)$ & 1.75 & 1.68 \\
\hline Eu II & 63 & 0.52 & $0.39(\operatorname{syn})$ & -0.13 & 0.36 & $0.13(1)$ & -0.39 & 0.34 & $0.74($ syn $)$ & 0.22 & 0.15 \\
\hline
\end{tabular}

Table A4 : Elemental abundances in HD 176021, HD 188985 and HD 202020

\begin{tabular}{|c|c|c|c|c|c|c|c|c|c|c|c|}
\hline & \multirow[b]{2}{*}{$\mathrm{Z}$} & \multirow[b]{2}{*}{ Solar $\log \epsilon^{a}$} & \multicolumn{3}{|c|}{ HD 176021} & \multicolumn{3}{|c|}{ HD 188985} & \multicolumn{3}{|c|}{ HD 202020} \\
\hline & & & $\log \epsilon$ & {$[\mathrm{X} / \mathrm{H}]$} & {$[\mathrm{X} / \mathrm{Fe}]$} & $\log \epsilon$ & {$[\mathrm{X} / \mathrm{H}]$} & {$[\mathrm{X} / \mathrm{Fe}]$} & $\log \epsilon$ & {$[\mathrm{X} / \mathrm{H}]$} & {$[\mathrm{X} / \mathrm{Fe}]$} \\
\hline $\mathrm{C}$ & 6 & 8.43 & 8.33(syn) & -0.10 & 0.52 & 8.61(syn) & 0.18 & 0.17 & $8.70($ syn $)$ & 0.27 & 0.23 \\
\hline $\mathrm{N}$ & 7 & 7.83 & 7.80 (syn) & -0.03 & 0.59 & $8.55(\mathrm{syn})$ & 0.72 & 0.71 & $8.44(\mathrm{syn})$ & 0.61 & 0.57 \\
\hline $\mathrm{O}$ & 8 & 8.69 & - & - & - & 8.66(syn) & -0.03 & -0.04 & $8.40(\mathrm{syn})$ & -0.29 & -0.33 \\
\hline $\mathrm{Na} \mathrm{I}$ & 11 & 6.24 & $5.80 \pm 0.01(2)$ & -0.44 & 0.18 & - & - & - & $6.35 \pm 0.06(3)$ & 0.11 & 0.07 \\
\hline $\mathrm{Mg} \mathrm{I}$ & 12 & 7.60 & $7.36 \pm 0.06(3)$ & -0.24 & 0.38 & $7.67 \pm 0.15(3)$ & 0.07 & 0.06 & $7.91 \pm 0.06(3)$ & 0.31 & 0.27 \\
\hline Si I & 14 & 7.51 & - & - & - & - & - & - & - & - & - \\
\hline $\mathrm{Ca} \mathrm{I}$ & 20 & 6.34 & $5.85 \pm 0.14(6)$ & -0.49 & 0.13 & $6.15 \pm 0.10(10)$ & -0.19 & -0.20 & $6.16 \pm 0.11(14)$ & -0.18 & -0.22 \\
\hline Sc II & 21 & 3.15 & $2.54(\mathrm{syn})$ & -0.61 & 0.01 & 2.95(syn) & -0.20 & -0.21 & 3.14(syn) & -0.01 & -0.05 \\
\hline Ti I & 22 & 4.95 & $4.62 \pm 0.09(3)$ & -0.33 & 0.29 & $4.90 \pm 0.10(4)$ & -0.05 & -0.06 & $5.11 \pm 0.09(7)$ & 0.16 & 0.12 \\
\hline Ti II & 22 & 4.95 & $4.72 \pm 0.12(2)$ & -0.23 & 0.42 & $4.83 \pm 0.14(3)$ & -0.12 & -0.13 & $5.05 \pm 0.09(6)$ & 0.10 & 0.02 \\
\hline V I & 23 & 3.93 & $2.88(\operatorname{syn})$ & -1.05 & -0.43 & - & - & - & 3.79 (syn) & -0.14 & -0.18 \\
\hline Cr I & 24 & 5.64 & $5.06 \pm 0.09(3)$ & -0.58 & 0.04 & $5.82 \pm 0.06(6)$ & 0.18 & 0.17 & $5.71 \pm 0.09(7)$ & 0.13 & 0.09 \\
\hline Cr II & 24 & 5.64 & $4.93 \pm 0.08(2)$ & -0.71 & 0.06 & - & - & - & - & - & - \\
\hline Mn I & 25 & 5.43 & $4.98(\operatorname{syn})$ & -0.45 & 0.17 & 5.06(syn) & -0.37 & -0.38 & 5.15(syn) & -0.28 & -0.32 \\
\hline Fe I & 26 & 7.50 & $6.88 \pm 0.08(37)$ & -0.62 & - & $7.51 \pm 0.10(45)$ & 0.01 & - & $7.54 \pm 0.09(120)$ & 0.04 & - \\
\hline Fe II & 26 & 7.50 & $6.84 \pm 0.05(2)$ & -0.65 & - & $7.51 \pm 0.09(16)$ & 0.01 & - & $7.58 \pm 0.03(4)$ & 0.08 & - \\
\hline Co I & 27 & 4.99 & $4.66(1)$ & -0.33 & 0.29 & $5.33(1)$ & 0.34 & 0.33 & $5.00 \pm 0.07(3)$ & 0.01 & -0.03 \\
\hline Ni I & 28 & 6.22 & $5.64 \pm 0.15(4)$ & -0.58 & 0.04 & $6.33 \pm 0.19(6)$ & 0.11 & 0.10 & $6.27 \pm 0.08(7)$ & 0.05 & 0.01 \\
\hline Zn I & 30 & 4.56 & $4.23 \pm 0.09(2)$ & -0.33 & 0.29 & $4.82 \pm 0.11(2)$ & 0.22 & 0.21 & $5.00 \pm 0.13(2)$ & 0.44 & 0.40 \\
\hline Sr I & 38 & 2.87 & 4.14(syn) & 1.27 & 1.89 & 4.95(syn) & 2.08 & 2.07 & 4.73(syn) & 1.86 & 1.82 \\
\hline Y II & 39 & 2.21 & $2.67 \pm 0.11(3)$ & 0.46 & 1.11 & $3.35 \pm 0.12(6)$ & 1.14 & 1.13 & $3.25 \pm 0.05(6)$ & 1.04 & 1.00 \\
\hline $\mathrm{Zr}$ II & 40 & 2.58 & - & - & - & - & - & - & $3.14(1)$ & 0.56 & 0.48 \\
\hline Ba II & 56 & 2.18 & $2.88($ syn $)$ & 0.70 & 1.35 & $3.65(\operatorname{syn})$ & 1.47 & 1.46 & $3.06($ syn $)$ & 0.88 & 0.80 \\
\hline La II & 57 & 1.10 & $1.78(\mathrm{syn})$ & 0.68 & 1.33 & $2.50(\mathrm{syn})$ & 1.40 & 1.39 & 1.93(syn) & 0.83 & 0.75 \\
\hline Ce II & 58 & 1.58 & $2.40 \pm 0.17(3)$ & 0.82 & 1.47 & $3.15 \pm 0.21(5)$ & 1.57 & 1.56 & $2.48 \pm 0.06(2)$ & 0.90 & 0.82 \\
\hline Pr II & 59 & 0.72 & - & - & - & - & - & - & - & - & - \\
\hline Nd II & 60 & 1.42 & $2.09 \pm 0.04(4)$ & 0.67 & 1.32 & $2.80 \pm 0.06(3)$ & 1.38 & 1.37 & $2.21 \pm 0.18(5)$ & 0.79 & 0.71 \\
\hline Sm II & 62 & 0.96 & $1.81 \pm 0.04(2)$ & 0.85 & 1.50 & - & - & - & - & - & - \\
\hline $\mathrm{Eu} \mathrm{II}$ & 63 & 0.52 & $0.25(\operatorname{syn})$ & -0.27 & 0.38 & $0.63($ syn $)$ & 0.11 & 0.10 & 0.67 (syn) & 0.15 & 0.07 \\
\hline
\end{tabular}

${ }^{a}$ Asplund (2009), The number inside the parenthesis shows the number of lines used for the abundance determination. 
Table A5: Equivalent widths (in $\mathrm{mÅ}$ ) of Fe lines used for deriving atmospheric parameters.

\begin{tabular}{|c|c|c|c|c|c|c|c|c|c|c|c|c|c|}
\hline Wavelength $(\AA)$ & Element & $E_{\text {low }}(\mathrm{eV})$ & $\log g f$ & CD-28 1082 & HD 29370 & CD-38 2151 & HD 50264 & HD 87080 & HD 123701 & HD 176021 & HD 188985 & HD 202020 & References \\
\hline 4114.446 & $\mathrm{Fe} I$ & 2.831 & -1.220 & - & - & - & - & - & - & - & $72.9(7.34)$ & - & 1 \\
\hline 4139.927 & & 0.990 & -3.629 & - & - & - & - & - & - & $43.5(6.81)$ & - & $67.4(7.58)$ & 1 \\
\hline 4184.891 & & 2.831 & -0.860 & - & - & - & - & - & - & - & $99.0(7.50)$ & - & 1 \\
\hline 4348.937 & & 2.990 & -2.130 & - & - & - & $41.0(7.20)$ & - & - & - & $35.6(7.31)$ & $48.7(7.39)$ & 1 \\
\hline 4566.514 & & 3.301 & -2.250 & - & - & - & $28.2(7.27)$ & - & - & - & - & $37.9(7.52)$ & 1 \\
\hline
\end{tabular}

The numbers in the parenthesis in columns 5-13 give the derived abundances from the respective lines. References: 1. Führ et al. (1988)

Note: This table is available in its entirety in online only. A portion is shown here for guidance regarding its form and content.

Table A6: Equivalent widths (in $\mathrm{mÅ}$ ) of Fe lines used for deriving atmospheric parameters.

\begin{tabular}{cccccccc}
\hline Wavelength $(\AA)$ & Element & $E_{\text {low }}(\mathrm{eV})$ & log gf & HD 30443 & HE 0308-1612 & HD 87853 & References \\
\hline 4114.446 & Fe I & 2.831 & -1.220 & - & - & - & 1 \\
4139.927 & & 0.990 & -3.629 & - & - & - & 1 \\
4147.669 & & 1.485 & -2.104 & - & - & $83.6(6.82)$ & 1 \\
4184.891 & & 2.831 & -0.860 & - & - & $83.8(6.79)$ & 1 \\
4187.039 & & 2.449 & -0.548 & - & - & $108.4(6.78)$ & 1 \\
\hline
\end{tabular}

The numbers in the parenthesis in columns 5-7 give the derived abundances from the respective line. References: 1. Führ et al. (1988)

Note: This table is available in its entirety in online only. A portion is shown here for guidance regarding its form and content.

Table A7: Lines used for deriving elemental abundances

\begin{tabular}{|c|c|c|c|c|c|c|c|c|c|c|c|c|c|}
\hline Wavelength $(\AA)$ & Element & $E_{\text {low }}(\mathrm{eV})$ & $\log g f$ & CD-28 1082 & HD 29370 & CD-38 2151 & HD 50264 & HD 87080 & HD 123701 & HD 176021 & HD 188985 & HD 202020 & Reference \\
\hline 5682.633 & $\mathrm{NaI}$ & 2.102 & -0.700 & - & $129.7(6.32)$ & - & - & $70.2(5.92)$ & - & $54.5(5.81)$ & - & $93.2(6.41)$ & 1 \\
\hline 5889.951 & & 0.000 & 0.100 & $166.7(4.56)$ & - & - & - & - & - & - & - & - & 1 \\
\hline 6154.226 & & 2.102 & -1.560 & - & - & - & - & - & $55.6(6.36)$ & - & - & $35.2(6.35)$ & 1 \\
\hline 6160.747 & & 2.104 & -1.260 & - & - & - & - & $30.2(5.79)$ & $76.8(6.38)$ & $24.2(5.79)$ & - & $48.4(6.28)$ & 1 \\
\hline 4571.096 & $\mathrm{Mg} \mathrm{I}$ & 0.000 & -5.691 & $56.6(5.89)$ & - & $170.4(6.64)$ & $92.9(7.60)$ & - & - & $86.1(7.42)$ & - & $108(7.95)$ & 2 \\
\hline 4730.029 & & 4.346 & -2.523 & - & - & - & $52.5(7.68)$ & - & - & - & $50.3(7.83)$ & 66.(7.95) & 3 \\
\hline
\end{tabular}

The numbers in the parenthesis in columns 5-13 give the derived abundances from the respective lines. References: 1. Kurucz et al. (1975), 2. Laughlin et al. (1974), 3. Lincke et al. (1971)

Note: This table is available in its entirety in online only. A portion is shown here for guidance regarding its form and content.

Table A8 : Lines used for deriving elemental abundances

\begin{tabular}{cccccccc}
\hline Wavelength $(\AA)$ & Element & $E_{\text {low }}(\mathrm{eV})$ & log gf & HD 30443 & HE 0308-1612 & HD 87853 & References \\
\hline 5682.633 & Na I & 2.102 & -0.700 & $99.20(4.96)$ & $108.9(6.00)$ & - & 1 \\
5688.205 & & 2.100 & -0.450 & $135.5(5.11)$ & $115.5(6.82)$ & - & 1 \\
5889.951 & & 0.000 & 0.100 & - & - & - & 1 \\
6154.226 & & 2.102 & -1.560 & - & - & - & 1 \\
6160.747 & & 2.104 & -1.260 & $86.60(5.36)$ & - & - & 1 \\
4571.096 & Mg I & 0.000 & -5.691 & - & - & - & 2 \\
4702.991 & Mg I & 4.346 & -0.666 & - & $177.7(7.22)$ & $142.2(7.55)$ & 3 \\
\hline
\end{tabular}

The numbers in the parenthesis in columns 5-7 give the derived abundances from the respective line.

References: 1. Kurucz et al. (1975), 2. Laughlin et al. (1974), 3. Lincke et al. (1971)

Note: This table is available in its entirety in online only. A portion is shown here for guidance regarding its form and content. 